\title{
New applications of renormalization group methods in nuclear physics
}

\author{
R.J. Furnstahl \\ Department of Physics, Ohio State University, Columbus OH 43210, USA \\ E-mail: furnstahl.1@osu.edu \\ K. Hebeler \\ Department of Physics, Ohio State University, Columbus OH 43210, USA \\ Institut für Kernphysik, Technische Universität Darmstadt, 64289 Darmstadt, \\ Germany \\ ExtreMe Matter Institute EMMI, GSI Helmholtzzentrum für Schwerionenforschung \\ GmbH, 64291 Darmstadt, Germany \\ E-mail: kai.hebeler@physik.tu-darmstadt.de
}

\begin{abstract}
We review recent developments in the use of renormalization group (RG) methods in low-energy nuclear physics. These advances include enhanced RG technology, particularly for three-nucleon forces, which greatly extends the reach and accuracy of microscopic calculations. We discuss new results for the nucleonic equation of state with applications to astrophysical systems such as neutron stars, new calculations of the structure and reactions of finite nuclei, and new explorations of correlations in nuclear systems.
\end{abstract}

\section{Contents}

1 Introduction 2

2 Renormalization group technology 7

2.1 Flow equations .................... 7

2.2 RG evolution of nucleon-nucleon interactions . . . . . . . . . . . . . . 8

2.3 RG evolution of three-nucleon interactions . . . . . . . . . . . . . . 11

2.4 Universality of low-momentum nuclear interactions . . . . . . . . . . . 14

3 Nuclear equation of state and astrophysical applications 15

3.1 Symmetric nuclear matter . . . . . . . . . . . . . . . . . 16

3.2 Neutron matter . . . . . . . . . . . . . . . . . . . . . . . 177

3.3 Applications to neutron stars . . . . . . . . . . . . . . 19 19

3.4 First results based on consistently evolved three-nucleon forces . . . . . . 20 


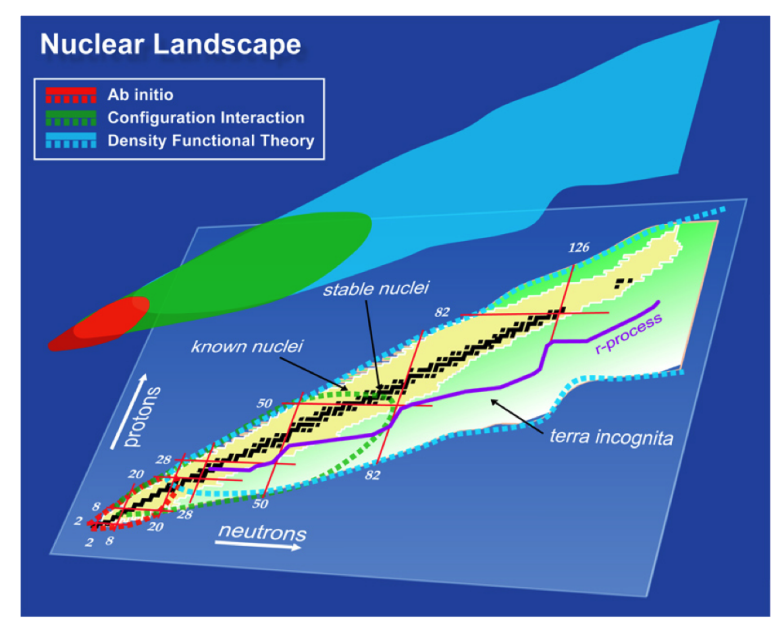

Figure 1. The nuclear landscape. A nuclide is specified by the number of protons and neutrons (figure from Ref. 2]). Most are unstable, e.g., to radioactive decay via the weak interaction. Fewer than half of the estimated total have been measured by experiment. The overlapping domains of theoretical methods are also indicated.

4 Applications to finite nuclei 21

4.1 Many-body perturbation theory in finite nuclei . . . . . . . . . . . . 21

4.2 Ab initio calculations with three-nucleon forces . . . . . . . . . . . 23

4.3 Ab-initio reactions with RG-evolved forces . . . . . . . . . . . . . . 24

4.4 In-medium Similarity Renormalization Group . . . . . . . . . . . . . . 27

4.5 Other applications and future directions ............. 31

5 Correlations in nuclear systems 32

5.1 Evolution of operators as an RG frontier . . . . . . . . . . . . . . 32

5.2 Scale dependence of short-range correlations . . . . . . . . . . . . 34

6 Summary and outlook

\section{Introduction}

The principal domain of low-energy nuclear physics is the table of the nuclides, shown in figure 1. There are several hundred stable nuclei (black squares) but also several thousand unstable nuclei are known through experimental measurements. However, the total number of nuclides is unknown (see the region marked "terra incognita"), with theoretical estimates suggesting approximately seven thousand total [1]. Many of these unstable nuclei ("rare isotopes") will be created and studied in new and planned experimental facilities around the world. An on-going challenge for low-energy nuclear theory is to describe the structure and reactions of all nuclei, whether measured or not.

A wide range of questions drive low-energy nuclear physics research [2]. At the fundamental level: How do protons and neutrons make stable nuclei and rare isotopes and where are the limits of nuclear existence? What is the equation of state of nucleonic 


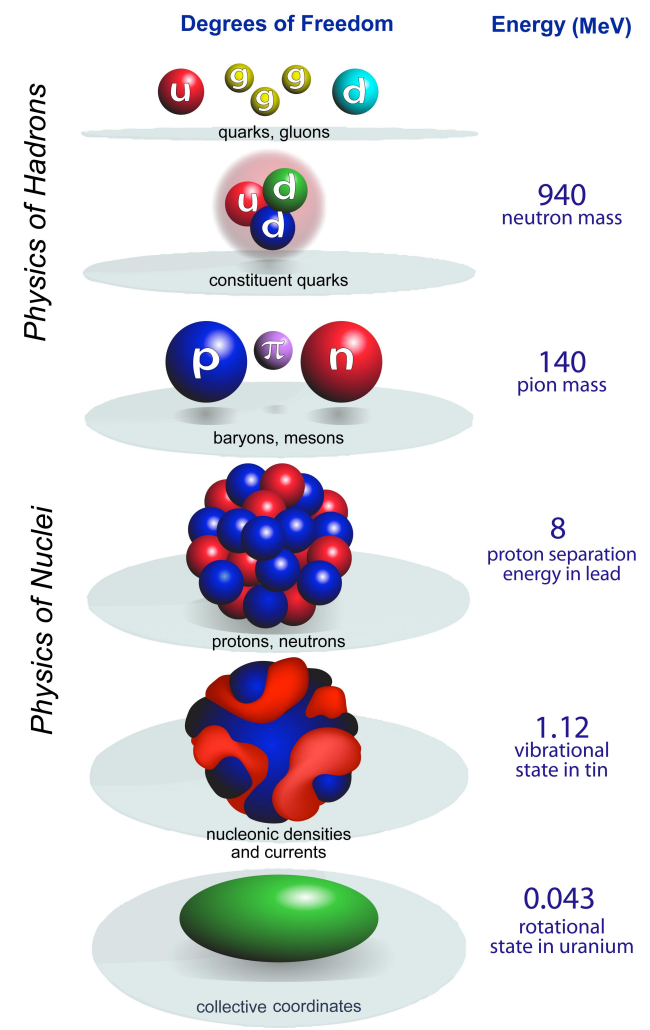

Figure 2. Hierarchy of nuclear degrees of freedom and associated energy scales [2].

matter? What is the origin of simple patterns observed in complex nuclei? How do we describe fission, fusion, and other nuclear reactions? These topics inform and are in turn illuminated by applications to other fields, such as astrophysics, where one can ask: How did the elements from iron to uranium originate? How do stars explode? What is the nature of neutron star matter? There are also connections to fundamental symmetries: Why is there now more matter than antimatter in the universe? What is the nature of the neutrinos, what are their masses, and how have they shaped the evolution of the universe? Finally, there are applications, for which we are led to ask: How can our knowledge of nuclei and our ability to produce them benefit humankind? The impact is very broad, encompassing the Life Sciences, Material Sciences, Nuclear Energy, and National Security.

In figure 2, the energy scales of nuclear physics are shown schematically. The extended hierarchy provides both challenges and opportunities. We can exploit the hierarchy by treating the ratio of scales as an expansion parameter, leading to a systematic, model-independent treatment at lower energies using effective field theory (EFT). The progression from top to bottom can be viewed as a reduction in resolution, which can be carried out theoretically using renormalization group (RG) methods. Our focus in this review is on the intermediate region only, where protons and neutrons are the relevant degrees of freedom. But even within this limited scope, the concept of 

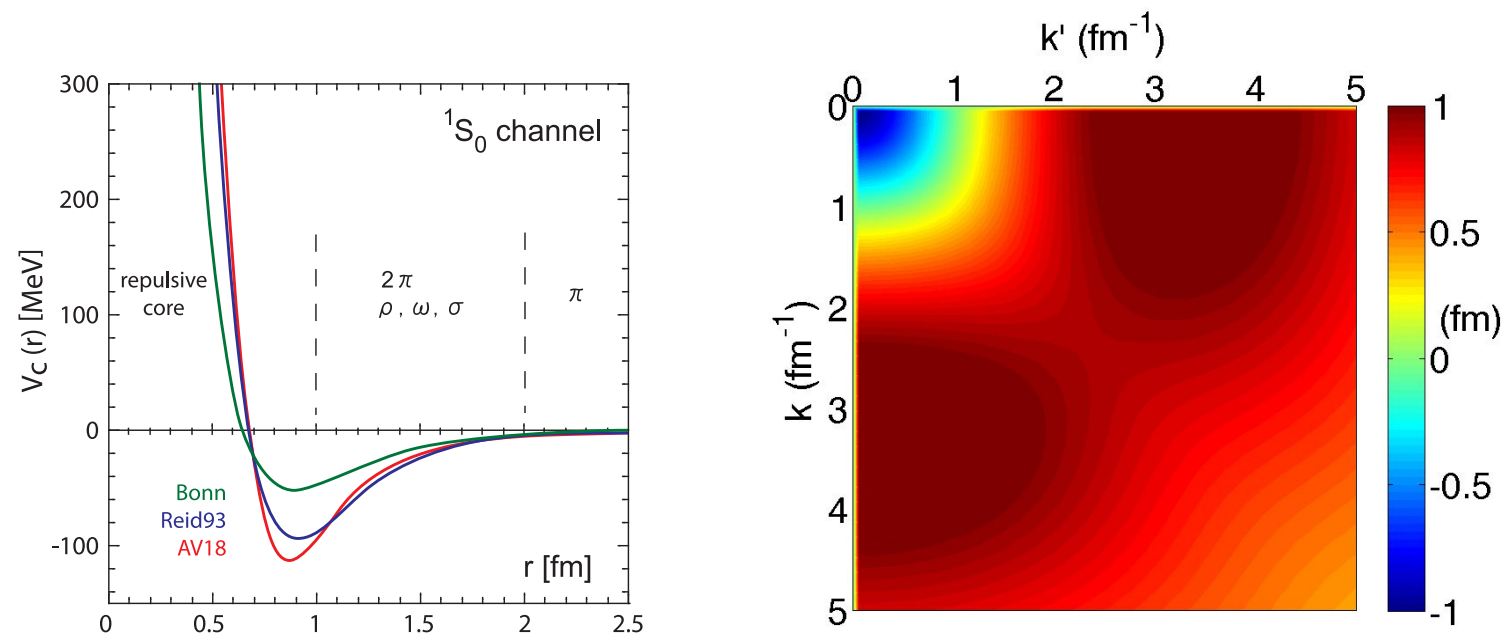

Figure 3. Left panel: three phenomenological potentials as functions of interparticle distance that accurately describe proton-neutron scattering up to laboratory energies of $300 \mathrm{MeV}$ [3. Right panel: alternative momentum space representation of the AV18 potential $\left\langle k\left|v_{18}\right| k^{\prime}\right\rangle$ in the ${ }^{1} \mathrm{~S}_{0}$ channel [4].

reducing resolution by $\mathrm{RG}$ methods is extremely powerful and fruitful.

The role of resolution scales can be illustrated using the phenomenon of diffraction: if the wavelength of light is comparable to or larger than an aperture, then diffraction is significant. Since two objects can only be resolved if the diffracted images do not overlap too much, the level of resolvable details depends crucially on the wavelength used. Being unable to resolve details at long wavelength is generally considered to be a disadvantage, but, as we will show in this review, it can be turned into an advantage.

A fundamental principle of any effective low-energy description is that if a system is probed at low energies, fine details are not resolved, and one can instead use low-energy variables. Short-distance structures can then be replaced by something simpler without affecting low-energy observables. This is analogous to using a truncated multipole expansion for a complicated charge or current distribution in classical electrodynamics. In the quantum case, EFT provides a systematic framework for such an expansion. We emphasize that while observable quantities do not change, the physics interpretation can (and generally does) change with resolution.

In many-body systems the natural resolution scale is set by the de Broglie wavelengths, i.e. by the typical momenta of the particles. By using units for which $\hbar=c=1$, the typical relative momentum in the Fermi sea of any large nucleus is of order $1 \mathrm{fm}^{-1}$ or $200 \mathrm{MeV}$. For our discussion, we will adopt $2 \mathrm{fm}^{-1}$ as the (arbitrary but reasonable) dividing line between low and high momentum for nuclei.

The left panel of figure 3 shows several phenomenological potentials as functions of the interparticle distance. Each was fit to reproduce nucleon-nucleon scattering phase shifts up to about $300 \mathrm{MeV}$ in lab energy. They are each characterized by a longrange attractive tail from one-pion exchange, intermediate attraction, and a strongly 


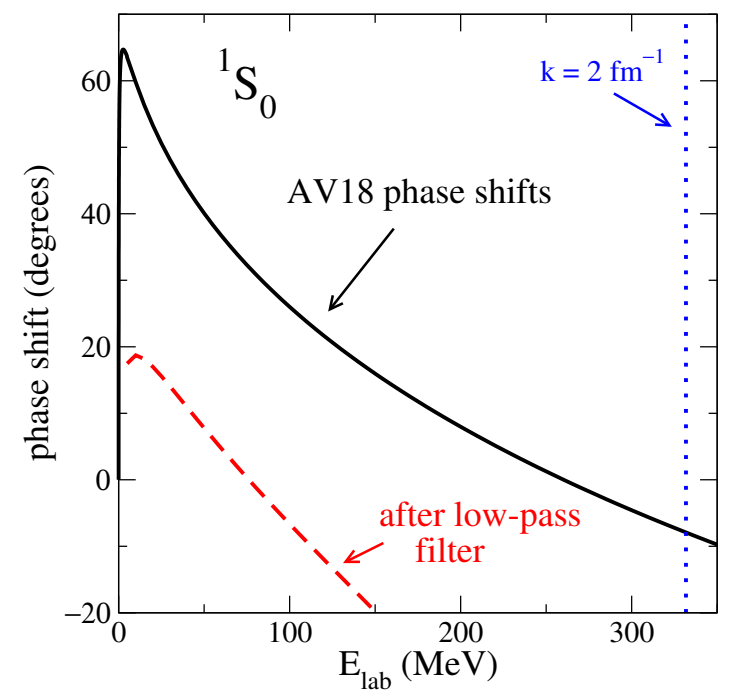

Figure 4. Effect of a low-pass filter on observables: the ${ }^{1} \mathrm{~S}_{0}$ phase shifts. The unaltered AV18 phase shifts reproduce experimentally extracted phase shifts in this energy range.

repulsive short-range "core". Alternatively, nuclear interactions can also be visualized in momentum space. In the right panel the potential AV18 is shown as a function of the initial and final relative momenta $k$ and $k^{\prime}$ of the two nucleons. Here the color coding indicates the strength and sign of the coupling of different momentum states.

It is evident from figure 3 that there are large matrix elements connecting low momenta with momenta much larger than $2 \mathrm{fm}^{-1}$. This is directly associated with the strong repulsive core of the potential. The consequences of these couplings can also be seen in wave functions and probability densities. Generally, these repulsive couplings lead to a significant suppression of the probability density at small separations. This suppression, called "short-range correlations" (SRC) in this context, significantly complicates basis expansions. For example, in a harmonic oscillator basis, which is frequently the choice for self-bound nuclei, convergence is substantially slowed by the need to accommodate these correlations. The factorial growth of the basis size with the number of nucleons then greatly limits the reach of calculations.

The underlying problem is that the resolution scale induced by the potential is mismatched with the scale of the low-energy nuclear states. This might seem to be analogous to compressing a digital photograph, which is readily accomplished by Fourier transforming and then applying a low-pass filter, i.e. simply setting the short wavelength parts to zero, and then transforming back. However, this strategy fails for nuclear potentials: figure 4 shows the results for the scattering phase shifts based on the original AV18 potential compared to the potential where all matrix elements for $k>2 \mathrm{fm}^{-1}$ have been set to zero. It is evident that the truncated potential fails completely to reproduce the phase shifts at all energies. The basic problem is that low and high momenta are coupled by the potential when solving quantum mechanically for observables.

Our solution to this problem is to decouple low and high energies rather than just 
setting the high-energy parts to zero. This can be achieved by a short-distance unitary transformation $U$. For example, for the evaluation of energy expectation values we can insert the relation $U^{\dagger} U=1$ twice:

$$
E_{n}=\left\langle\Psi_{n}|H| \Psi_{n}\right\rangle=\left\langle\Psi_{n}\left|U^{\dagger} U H U^{\dagger} U\right| \Psi_{n}\right\rangle \equiv\left\langle\widetilde{\Psi}_{n}\left|\widetilde{H}_{\mid}\right| \widetilde{\Psi}_{n}\right\rangle .
$$

with $\widetilde{H}=U H U^{\dagger}$ and $\left|\widetilde{\Psi}_{n}\right\rangle=U\left|\Psi_{n}\right\rangle$. In doing so operators and wavefunctions get modified but observables remain unchanged. An appropriate choice of the unitary transformation can, in principle, achieve the desired decoupling. This general approach has long been used in nuclear structure physics and for other many-body applications [5, 6, 7]. The new feature here is the use of RG flow equations to create the unitary transformation successively via a series of infinitesimal transformations.

The RG is well suited to this purpose and is more powerful and versatile than many other approaches. The common features of RG for critical phenomena and high-energy scattering are discussed by Steven Weinberg in an essay in Ref. [8]. He summarizes:

"The method in its most general form can I think be understood as a way to arrange in various theories that the degrees of freedom that you're talking about are the relevant degrees of freedom for the problem at hand."

This is the essence of what is done with the low-momentum interaction approaches considered here: arrange for the degrees of freedom for nuclear structure to be the relevant ones. This does not mean that other degrees of freedom cannot be used, but to again quote Weinberg [8]:

"You can use any degrees of freedom you want, but if you use the wrong ones, you'll be sorry."

The consequences of using RG for high-energy (particle) physics include improving perturbation theory, e.g., in QCD. A mismatch of energy scales can generate large logarithms that ruins perturbative convergence even when couplings by themselves are small. The RG shifts strength between loop integrals and coupling constants to reduce these logs. For critical phenomena in condensed matter systems, the RG reveals the nature of observed universal behavior by filtering out short-distance degrees of freedom.

We see both these aspects in our applications of RG to nuclear structure and reactions. As the resolution is lowered, nuclear calculations become more perturbative (e.g., see figures 7 and 21) and the potentials flow toward universal form (e.g., see figure 10. The end result can be said to make nuclear physics look more like quantum chemistry calculationally, opening the door to a wider variety of techniques (such as many-body perturbation theory) and simplifying calculations (e.g., by improving convergence of basis expansions). On the other hand, microscopic three-nucleon forces $(3 \mathrm{NF})$ have been found to be essential for accurate results, and developing RG technology to handle them is an on-going challenge.

Over the last decade there have been increasing applications of RG technology to low-energy physics. This brief review focuses on the most recent developments and therefore details of prior work are necessarily limited. More extensive reviews 

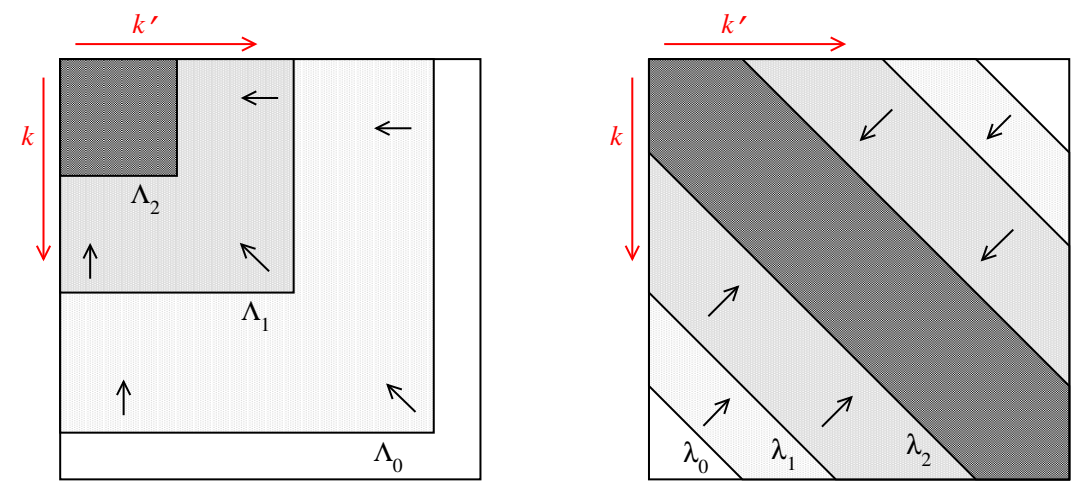

Figure 5. Schematic illustration of two types of RG evolution for NN potentials in momentum space: (a) $V_{\text {low } k}$ running in $\Lambda$, and (b) SRG running in $\lambda$ for $G_{s}=T$ (see main text). Here $k\left(k^{\prime}\right)$ denote the relative momenta of the initial (final) state. At each $\Lambda_{i}$ or $\lambda_{i}$, the matrix elements outside of the corresponding lines are negligible, so that high- and low-momentum states are decoupled.

of the earlier progress can be found in Refs. 4] and 9] and references therein. In Section 2 we present the basics of RG technology for evolving two- and three-body nuclear potentials, concentrating on new developments. Our starting potentials are generally taken from chiral EFT, which provides a systematic hierarchy of initial twoand higher-body interactions. Recent progress in calculating the equation of state of nucleonic matter with applications to neutron stars is discussed in Section 3 . There are many new results for both the structure and reactions of finite nuclei, which are reviewed in Section 4. In Section 5 we discuss the use of RG with external probes of nuclear correlations. We conclude in Section 6 with a summary of the main points, on-going developments, and important open questions.

\section{Renormalization group technology}

In this section, we give a brief overview of the equations and techniques used to derive low-momentum nuclear interactions. We illustrate how RG methods decouple low- and high-energy degrees of freedom and how this leads to simplified many-body calculations. We emphasize recent developments, such as the evolution of many-body forces, the use of local projections for visualization of interactions, and the phenomenon of universality in nuclear many-body forces at low resolution.

\subsection{Flow equations}

At the heart of every RG framework is a flow equation. In general, the flow equation is a set of coupled differential equations for operators and couplings. In low-energy nuclear physics, these equations specify how matrix elements of the nuclear interactions change when the $\mathrm{RG}$ resolution scale is varied by an infinitesimal amount. In figure 5 , two common options are shown for how the RG can be used to decouple a two-body 
Hamiltonian. As in figure $3, k\left(k^{\prime}\right)$ denotes the initial (final) relative momentum of the interacting nucleons. The more traditional approach in the left panel lowers a momentum cutoff $\Lambda$ in small steps, with the matrix elements adjusted by requiring some observables such as nucleon-nucleon scattering phase shifts remain invariant up to the momentum scale $\Lambda$. Matrix elements well above $\Lambda$ are zero and are therefore trivially decoupled. In low-energy nuclear physics this approach is typically referred to

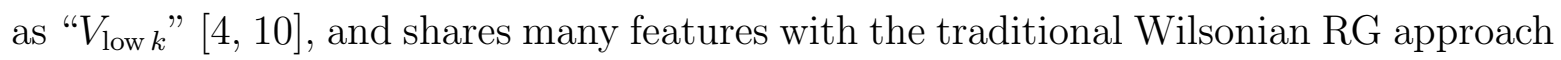
in field theory, corresponding to successively integrating out "momentum shells" [11]. In recent years this $V_{\text {low } k}$ approach has been applied very successfully to various twonucleon forces [4]. However, as discussed in Section 1, three- and higher-body forces have been shown to play an important role in nuclear systems and there are unsolved technical complications in systematically treating such many-body forces within the $V_{\text {low } k}$ framework.

A more recent $R G$ approach to the nuclear Hamiltonian is illustrated in the right panel of figure 5, in which the matrix is driven toward band-diagonal form to achieve decoupling of low- and high momenta. This RG framework was originally developed in the early 1990's by Wegner [12, 13, 14] for condensed matter applications under the name "Hamiltonian flow equations" and independently by Glazek and Wilson [15] for solving quantum chromodynamics in light-front formalism under the name "similarity renormalization group" (SRG). Only in the last five years was it realized that this approach is particularly well suited for low-energy nuclear physics, where it is technically simpler and more versatile than other methods such as the $V_{\text {low } k}$ approach [4, 16].

The basic idea of the SRG is to apply a unitary transformation as in Eq. (1) to an initial Hamiltonian, $H=T_{\text {rel }}+V$, in a series of infinitesimal steps, labeled by the flow parameter $s$ increasing from $s=0$ :

$$
H_{s}=U_{s} H U_{s}^{\dagger} \equiv T_{\text {rel }}+V_{s}
$$

with $U_{s}^{\dagger} U_{s}=U_{s} U_{s}^{\dagger}=1$. Here, $T_{\text {rel }}$ is the relative kinetic energy operator, which is chosen to be invariant under the unitary transformation and $V_{s}$ indicates all two-nucleon and higher-body interactions. Any unitary transformation can be recast in form of a flow equation [16]:

$$
\frac{d H_{s}}{d s}=\left[\eta_{s}, H_{s}\right] \quad \text { with } \quad \eta_{s} \equiv \frac{d U_{s}}{d s} U_{s}^{\dagger}
$$

The anti-Hermitian generator $\eta_{s}$ can be specified by a commutator of $H_{s}$ with a Hermitian operator $G_{s}$, i.e. $\eta_{s}=\left[G_{s}, H_{s}\right]$, to obtain a transformation that tends to diagonalize $H_{s}$ in the eigenbasis of $G_{s}$.

\section{2. $R G$ evolution of nucleon-nucleon interactions}

The operator $G_{s}$ completely defines the flow of a given initial Hamiltonian and there are many possible choices one can consider. The most common choice in recent applications for its simplicity and effectiveness is $G_{s}=T_{\text {rel }}$. The evolution of a two-body force as $s$ increases or as $\lambda \equiv s^{-1 / 4}$ decreases is illustrated for this case schematically in the right 


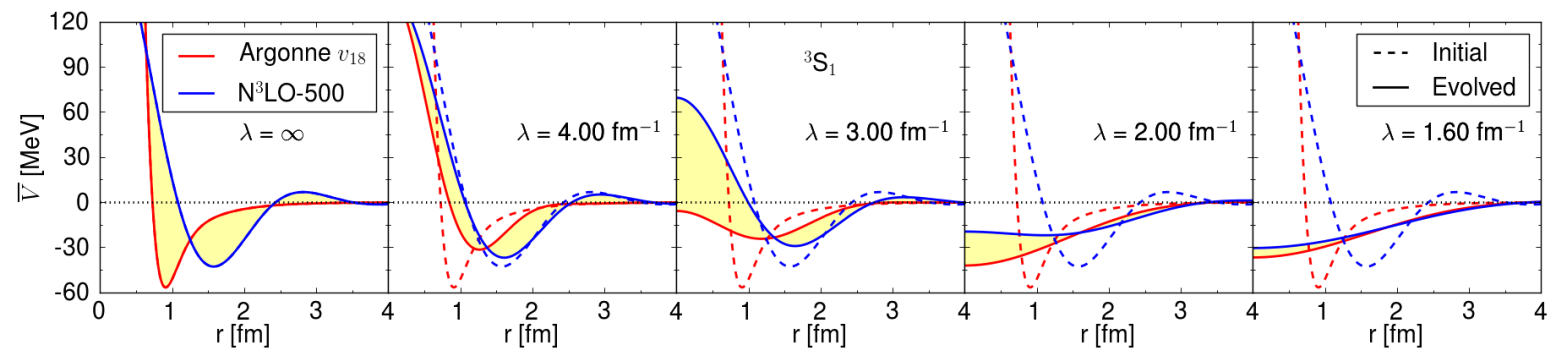

Figure 6. Local projection of $\mathrm{AV} 18$ and $\mathrm{N} 3 \mathrm{LO}(500 \mathrm{MeV})$ potentials in ${ }^{3} \mathrm{~S}_{1}$ channel at different resolutions [17. The dashed lines show the matrix elements of the initial unevolved potentials.

panel of figure 5. The off-diagonal strength gets successively suppressed and the matrix is driven towards a band-diagonal form with $\lambda$ a measure of the degree of decoupling (see [18, 19] for exceptions). During the RG evolution the low-energy physics is shifted to the low-momentum part of the Hamiltonian and other observables. The preservation of the low-energy physics at all scales under the RG flow is guaranteed by the unitarity of the transformation $U_{s}$.

The effects of the RG evolution can also be visualized in configuration space. Since SRG-evolved interactions are in general non-local, i.e. non-diagonal in coordinate representation, it is convenient to consider for visualization purposes a local projection of the nuclear potentials (given here for S-waves) [17]:

$$
\bar{V}_{\lambda}(r)=\int_{0}^{\infty} r^{\prime 2} d r^{\prime} V_{\lambda}\left(r, r^{\prime}\right)
$$

The local projections for two realistic NN potentials at different SRG resolution scales are shown in figure 6. As already shown in figure 3, the potential "AV18" shows a very strong repulsive short-range part at high resolution scales (left panel). The potential " $\mathrm{N}^{3} \mathrm{LO}$ " denotes a very commonly used high-precision potential which has been derived within chiral EFT [20]. For this potential the short-range repulsion is much less pronounced, but still significant. Clearly, during the flow to lower scales the short-range repulsive parts get gradually dissolved [17]. Also evident is the flow of the two potentials, initially quite different, toward a universal form at the lower values of $\lambda$. This phenomenon is referred to as "universality" of nucleon-nucleon forces at low resolution and will be discussed in more detail below.

Besides the canonical choice $G_{s}=T_{\text {rel }}$, it is also possible to choose a generator that reproduces the block diagonal (as opposed to band diagonal) form of the $V_{\text {low } k}$ RG shown schematically in figure 5, except that the transformation will be unitary [21]. Recently, alternative generators that allow computationally much faster evolution have been explored, see Ref. 22 for details.

The evolution to lower resolution scales is accompanied by a shift of physics. In particular, effects of short-range two-body interactions are replaced by new longerrange two- and many-body forces. We emphasize that the relative importance of 


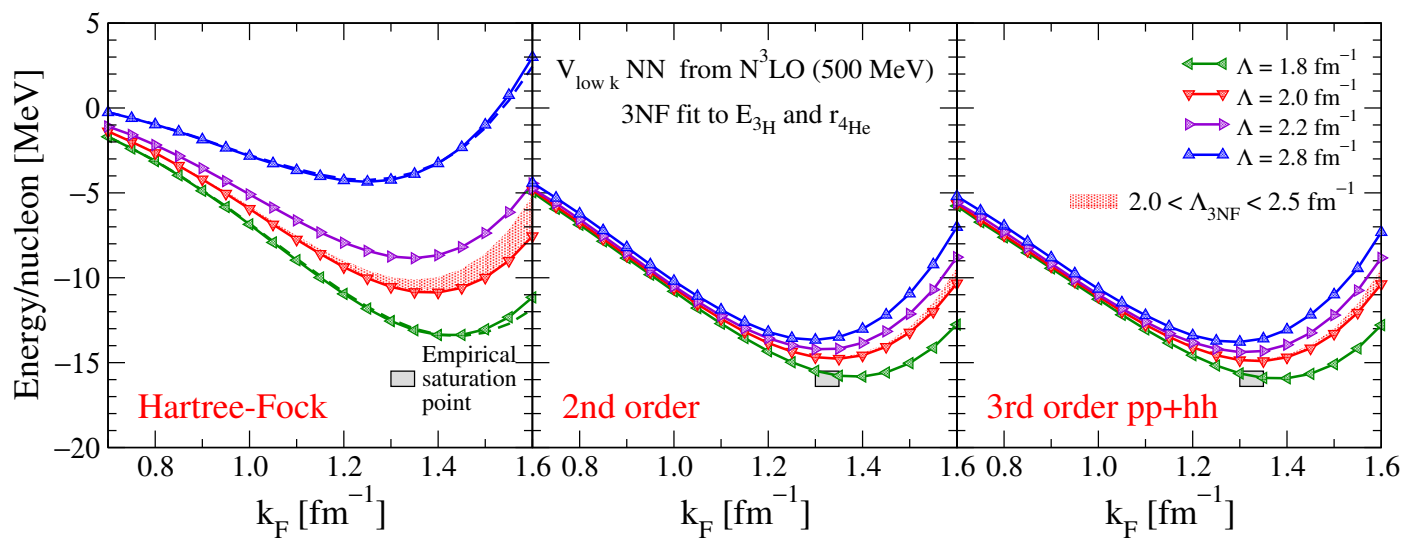

Figure 7. Nuclear matter energy per particle versus Fermi momentum $k_{\mathrm{F}}$ at the Hartree-Fock level (left) and including second-order (middle) and third-order particleparticle/hole-hole contributions (right), based on evolved $\mathrm{N}^{3} \mathrm{LO} \mathrm{NN}$ potentials and $3 \mathrm{NF}$ fit to $E_{3_{3}}$ and $r_{4} \mathrm{He}$. Theoretical uncertainties are estimated by the NN (lines) $/ 3 \mathrm{~N}$ (band) cutoff variations. See Ref. 23] for details.

separate contributions to observables from different sectors of the Hamiltonian or from different orders in perturbation theory are resolution dependent and consequently are not themselves observables.

We illustrate the improved perturbativeness at low resolution scales using results for infinite nuclear matter. We will discuss the physics of nuclear matter and its relevance for astrophysical applications in more detail in Section 3. Here, we only illustrate the convergence pattern of the many-body expansion as a function of the RG resolution scale. In figure 7 the energy per particle of symmetric nuclear matter is shown as a function of Fermi momentum $k_{\mathrm{F}}$, with density $\rho=2 k_{\mathrm{F}}^{3} /\left(3 \pi^{2}\right)$. The grey square represents the empirical saturation point in each panel. Its boundaries reflect the ranges of nuclear matter saturation properties predicted by phenomenological Skyrme energy functionals that most accurately reproduce properties of finite nuclei [24]. The figure shows results based on $V_{\text {low }} k^{\text {-evolved }} \mathrm{NN}$ interactions plus contributions from $3 \mathrm{~N}$ interactions in three many-body approximations: Hartree-Fock (left), Hartree-Fock plus second-order contributions (middle), and additionally summing selected third-order contributions (right). Evidently, the size of the higher-order contributions in the manybody expansion become smaller with decreasing cutoff $\Lambda$; for all values considered the third-order diagrams provide only very small contributions.

A calculation without approximations should be independent of the RG resolution scale. In practice, there will be approximations both in the implementation of the RG and in the subsequent calculations of nuclear structure observables. That is, cutoff dependence arises because of the truncation of induced many-body forces (see below) or because of many-body approximations. Hence it is possible to use the cutoff-dependence as a diagnostic of approximations and to estimate theoretical errors. For example, in figure 7 the second-order results show a significant narrowing of the spread over a 


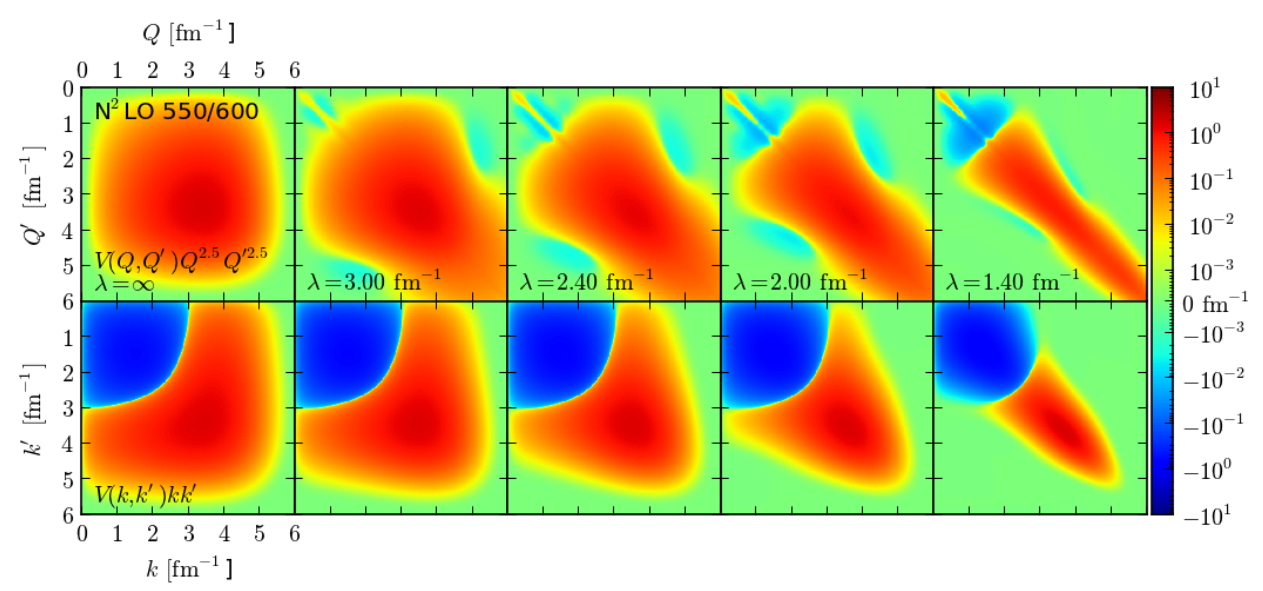

Figure 8. Contour plot of the $3 \mathrm{NF}$ (upper row) and $2 \mathrm{NF}$ (lower row) as a function of $\lambda$ for the $550 / 600 \mathrm{MeV}$ chiral EFT potential. The lowest antisymmetric hyperspherical partial wave is plotted as well as the two-body partial waves that are embedded in this three-body partial wave. See Ref. [26] for details.

large density region compared to Hartree-Fock results. The observed remaining cutoff dependence in the right panel provides a scale for neglected four-body interactions. Indeed, this size is consistent with the expected size based on chiral EFT [25] (see Section 3).

\subsection{RG evolution of three-nucleon interactions}

When evolving nuclear interactions to lower resolution, it is inevitable that manybody interactions and operators are induced even if initially absent [27]. This might be considered problematic if nuclei could be accurately calculated based on only NN interactions, as was assumed for much of the history of nuclear structure calculations. However, chiral EFT has revealed a natural scale and hierarchy of many-body forces, which dictates that more than NN be included in modern calculations of nuclei and nucleonic matter [28, 29]. Thus, the real concern is whether this hierarchy is maintained as nuclear interactions are evolved (see Section 3). The consistent treatment of threebody forces in the RG evolution and in many-body calculations is a complex task and currently one of the key frontiers in nuclear physics.

Currently, there exist different ways to treat $3 \mathrm{~N}$ forces in the $\mathrm{RG}$ framework:

(a) Starting from nuclear $\mathrm{NN}$ and $3 \mathrm{~N}$ forces, derived and fitted in chiral EFT, it is possible to systematically evolve the full Hamiltonian. For calculations of light and medium mass nuclei this has been achieved by representing Eq. (3) using a discrete harmonic oscillator basis [27]. Results for light nuclei based on this approach are very promising [30, 31]. For heavier nuclei however, significant scale dependencies have been found [31, 32] (see also Section 4), which suggest that infinite matter will not be realistic. These could be indications of significant induced $4 \mathrm{~N}$ forces or possibly an insufficient evolution of $3 \mathrm{~N}$ forces due to basis truncations. 


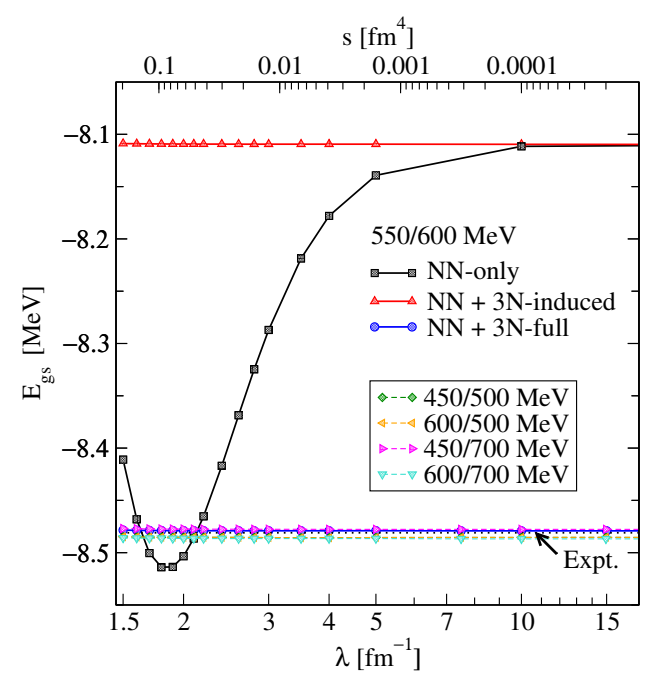

Figure 9. Ground state energy of ${ }^{3} \mathrm{H}$ as a function of the flow parameter $\lambda=s^{-1 / 4}$ for different initial chiral interactions. NN-only means initial and induced $3 \mathrm{~N}$ interactions are discarded, $\mathrm{NN}+3 \mathrm{~N}$-induced takes only induced $3 \mathrm{~N}$ interactions into account, and $3 \mathrm{~N}$-full contains initial and induces $3 \mathrm{~N}$ interactions. The black dotted line shows the experimental binding energy.

(b) Only the NN interactions are evolved with RG methods and then the chiral EFT $\mathrm{N}^{2} \mathrm{LO} 3 \mathrm{~N}$ force is added with its short-range parameters determined at the lowmomentum scale from fits to few-body systems. This procedure assumes that the long-range part of the $3 \mathrm{~N}$ forces remains invariant under the $\mathrm{RG}$ transformations and that the $\mathrm{N}^{2} \mathrm{LO}$ operator structure is a sufficiently complete operator basis that induced contributions can be absorbed to good approximation. The results shown in figure 7 are based on this strategy (and also Refs. [33, 34]), and are found to be in agreement with nuclear phenomenology within the theoretical uncertainties [23, 35].

(c) Recently a complementary framework to consistently evolve $3 \mathrm{~N}$ forces in a continuous plane-wave basis has been developed [36]. In this approach the evolution of $\mathrm{NN}$ and $3 \mathrm{~N}$ forces is separated explicitly, which allows the subtraction of $\mathrm{NN}$ interactions in a three-body basis to be avoided. Such momentum-space interactions can be directly used for calculations of infinite systems and finite nuclei. First results for neutron matter based on such interactions are presented in Section 3 . Since SRG transformations are usually characterized by the coupling patterns of momentum eigenstates, the momentum basis is a natural basis in which to construct the SRG generator $\eta_{s}$. The construction of optimized generators for suppressing the growth of many-body forces is currently under active investigation.

(d) Finally, there is a new framework for evolving $\mathrm{NN}$ and $3 \mathrm{~N}$ forces in a hyperspherical momentum representation [26]. This framework represents a hybrid approach in the sense that it is based on a continuous momentum basis like (c), but the RG evolution is performed for the entire Hamiltonian as in (a). The hyperspherical basis is particularly useful for visualizing matrix elements of interactions. In 


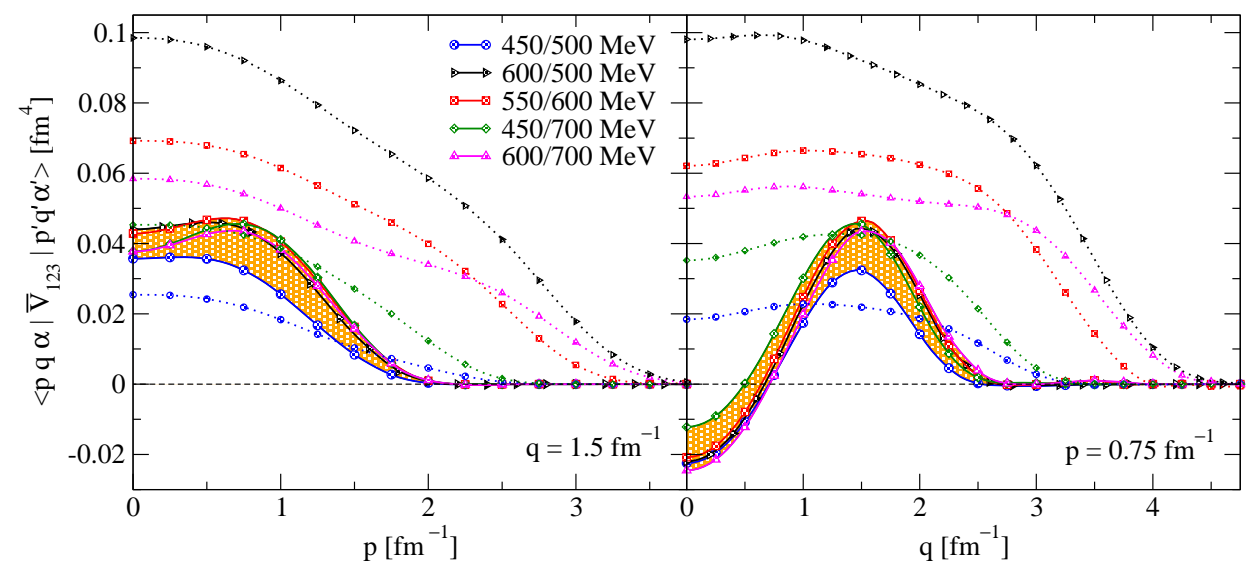

Figure 10. Matrix elements of the initial 3N forces (dotted lines) compared to evolved $3 \mathrm{~N}$ forces (solid lines) at $\lambda=1.5 \mathrm{fm}^{-1}$ for different interactions labeled by the values of the cutoffs $\Lambda / \tilde{\Lambda}$ [37. For details see Ref. [36]. The shaded band marks the maximal variation between different evolved $3 \mathrm{~N}$ force matrix elements.

figure 8 are shown some representative matrix elements of $\mathrm{NN}$ (bottom) and $3 \mathrm{~N}$ (top) interactions at different resolution scales. It is evident that both display the characteristic SRG decoupling pattern as they are evolved to lower scales.

To illustrate the basic ideas behind evolving three-body forces within the SRG framework for $G_{s}=T_{\text {rel }}$, we adopt a notation in which $V_{12}$ means the two-body interaction between particles 1 and 2 while $V_{123}$ is the irreducible three-body potential. We start with the Hamiltonian in the three-particle space:

$$
H_{s}=T_{\text {rel }}+V_{12}+V_{13}+V_{23}+V_{123} \equiv T_{\text {rel }}+V_{s} .
$$

The SRG flow equation (3) in this space is

$$
\frac{d H_{s}}{d s}=\frac{d V_{s}}{d s}=\frac{d V_{12}}{d s}+\frac{d V_{13}}{d s}+\frac{d V_{23}}{d s}+\frac{d V_{123}}{d s}=\left[\left[T_{\mathrm{rel}}, V_{s}\right], H_{s}\right],
$$

where $d H_{s} / d s=d V_{s} / d s$ because we define $T_{\text {rel }}$ to be independent of $s$. The equations for each of the two-body potentials (which are completely determined by their evolved matrix elements in the two-particle space) are

$$
\frac{d V_{12}}{d s}=\left[\left[T_{12}, V_{12}\right], T_{12}+V_{12}\right],
$$

were $T_{12}$ denotes the relative kinetic energy of particle 1 and 2 , and similarly for the $V_{13}$ and $V_{23}$ equations. When Eq. (7) is used in Eq. (6), particle 3 is a spectator. If we use a discrete basis this is not a problem, and it is straightforward to represent the Hamiltonian as a matrix and evolve the entire matrix in a three-body basis via Eq. (6). But in a continuous basis, delta functions associated with spectator particles in two-body interaction processes make this representation problematic. However, it is straightforward to show that the derivatives of two-body potentials on the left side cancel precisely with terms on the right side, leaving an explicit equation for evolving 
the three-body interaction separately from the two-body interaction [16],

$$
\begin{aligned}
\frac{d V_{123}}{d s} & =\left[\left[T_{12}, V_{12}\right], V_{13}+V_{23}+V_{123}\right]+\left[\left[T_{13}, V_{13}\right], V_{12}+V_{23}+V_{123}\right] \\
& +\left[\left[T_{23}, V_{23}\right], V_{12}+V_{13}+V_{123}\right]+\left[\left[T_{\text {rel }}, V_{123}\right], H_{s}\right] .
\end{aligned}
$$

The cancellations eliminate the disconnected spectator contributions (all 3 indices appear in each term on the right side) and the "dangerous" delta functions, so Eq. (8) can now be solved directly in a continuous basis. From this equation it is manifest that the matrix elements of $V_{123}$ will change even if initially $V_{123}=0$ on the right side.

In figure 9 we show for illustration the ground state energy of the triton for different initial chiral interactions at different SRG resolution scales based on method (b) (very similar results have been found using strategies (a) [27] and (d) [26]). For one interaction we show three different cases: "NN-only" corresponds to taking only NN interactions into account and discard all $3 \mathrm{~N}$ contributions, in the case "NN+3N-induced" we start with only NN forces at $\lambda=\infty$ but we keep all $3 \mathrm{~N}$ contributions which are "induced" during the SRG evolution and finally for "NN+3N-full" we include in addition $3 \mathrm{~N}$ interactions at $\lambda=\infty$ and retain all induced $3 \mathrm{~N}$ contributions during the RG flow. Evidently, neglecting induced $3 \mathrm{~N}$ forces results in a significant variation of the binding energy. Only after retaining consistently all $3 \mathrm{~N}$ contributions does the binding energy remain invariant under changes in the SRG resolution scale. Of course, this strict invariance holds only for three-body systems since neglected higher-body forces cannot contribute. It is one of the main frontiers to understand the nature and importance of many-body forces in four- and higher-body systems.

\subsection{Universality of low-momentum nuclear interactions}

As already illustrated in figure 6, low-resolution $\mathrm{NN}$ interactions are found to be quantitatively very similar [4, 16]. This universality can be attributed to common longrange pion physics and phase-shift equivalence of all potentials, which is reflected in the matrix elements at low resolution. It has been an open question whether the same is true for $3 \mathrm{~N}$ forces since there are important differences: First, chiral $3 \mathrm{~N}$ forces are fixed by fitting only two low-energy constants $\left(c_{D}\right.$ and $\left.c_{E}\right)$, in contrast to numerous couplings in NN interactions [28. Second, $3 \mathrm{~N}$ forces give only subleading contributions to observables. Since universality is only approximate in NN interactions, it is not obvious to what extent $3 \mathrm{~N}$ forces are constrained by long-range physics at low resolution. In figure 10 we illustrate explicitly the form of the matrix elements of $3 \mathrm{~N}$ forces at two resolution scales for five different chiral interactions (see Ref. [26] for another example of 3NF universality). We also find a remarkably reduced model dependence for evolved $3 \mathrm{~N}$ interactions in the dominant kinematical region. This suggests that the chiral low-energy coupling constants $c_{D}$ and $c_{E}$ are flowing to an approximately universal value at low resolution. In addition, new momentum-dependent universal structures are induced at low resolution, as can be seen in the right panel. Future plans involve the explicit extraction of the low-energy constants from the evolved matrix elements 

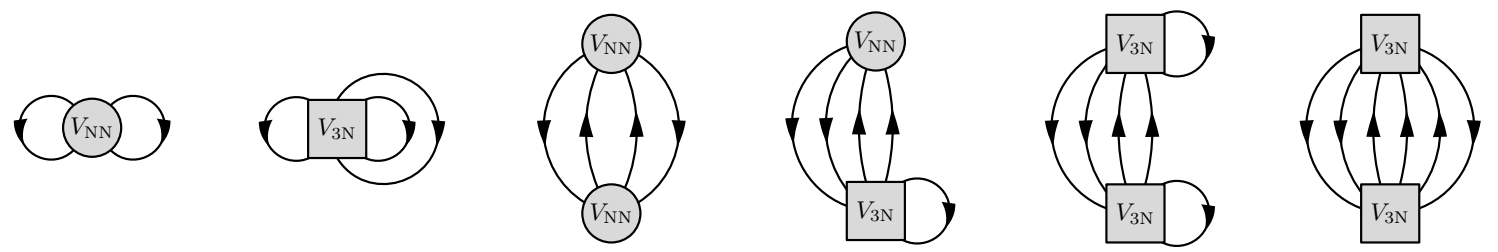

Figure 11. Diagrams contributing to the energy per particle up to second order in MBPT, taking two- and three-body interactions into account.

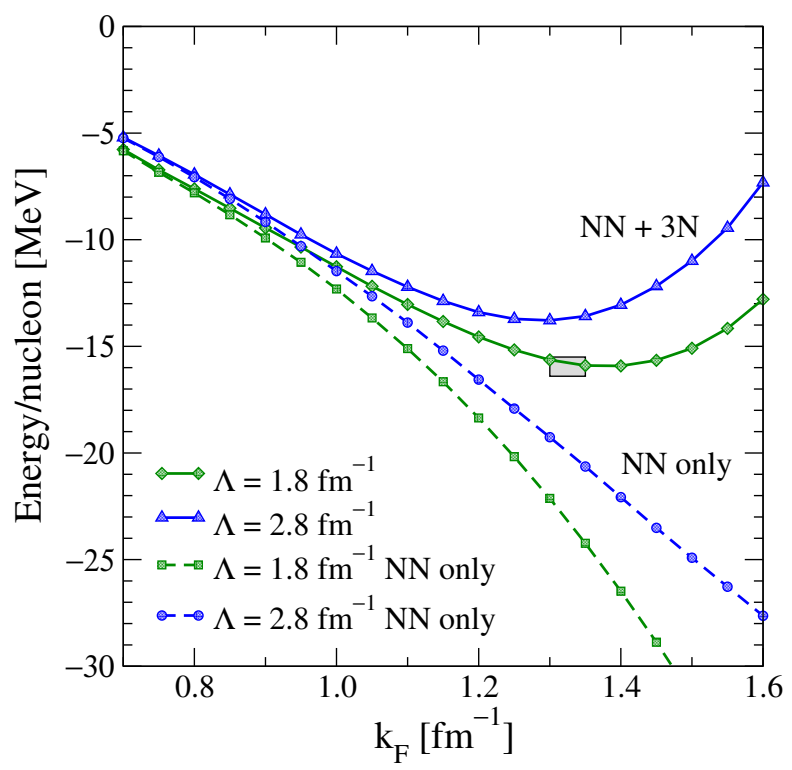

Figure 12. Energy per particle in symmetric nuclear matter as a function of the Fermi momentum based on NN+3NF forces compared to NN-only results for two representative NN cutoffs and a fixed 3N cutoff. For details see Ref. 23.

and the investigation of $3 \mathrm{NF}$ universality, in particular the role of the contributions at $\mathrm{N}^{3} \mathrm{LO}$ [38, 39].

\section{Nuclear equation of state and astrophysical applications}

Nuclear matter describes an idealized infinite system consisting of neutrons and protons in the thermodynamic limit, interacting only via the strong nuclear force and neglecting the Coulomb forces between protons. Neutron matter in particular consists only of neutrons, whereas symmetric nuclear matter refers to the special case with equal neutron and proton densities. The interplay of chiral EFT and RG methods offers new opportunities for efficient and simplified microscopic calculations of the nuclear equation of state. RG-evolved interactions enable the application of perturbative methods, which also provide improved estimates of theoretical uncertainties (see also the discussion of in-medium chiral perturbation theory in Ref. [40].) Figure 11 shows all interaction diagrams contributing to the energy up to second order in many-body perturbation 
theory (MBPT), taking two- and three-body interactions into account. These diagrams have been found to already provide well-converged results for the energy at small resolution scales (a nonperturbative validation of MBPT for two-body interactions in neutron matter has recently been given in [41]).

The physics of nuclear matter covers a wide range of extremes. At very low densities, the interparticle distances are sufficiently large that details of the nuclear interaction are not resolved and all properties of the system are governed by the large s-wave scattering length. In this universal regime neutron matter shares many features with properties of atomic gases close to the unitary limit, which are currently the subject of active theoretical and experimental investigations [42]. At intermediate densities, which are most relevant for finite nuclei, nuclear matter properties are used to guide the development of nuclear energy density functionals and in particular to constrain the physics of neutron-rich nuclei close to the limit of stability, which are key for understanding the synthesis of heavy nuclei in the universe. At very high densities, the composition and properties of nuclear matter are still unknown. Exotic states of matter containing strange particles or isolated quarks might be present under such conditions and possibly exist in the interior of neutron stars [43].

\subsection{Symmetric nuclear matter}

Over the last decades, an accurate prediction of symmetric nuclear matter at intermediate densities starting from microscopic nuclear forces has been a theoretical milestone on the way to finite nuclei close to the valley of stability, but has proved to be an elusive target. Progress for controlled calculations has long been hindered by the difficulty and the non-perturbative nature of the nuclear many-body problem when conventional nuclear interactions are used.

Most advances in microscopic nuclear structure theory over the last decade have been through expanding the reach of few-body calculations. This has clearly established the quantitative role of $3 \mathrm{~N}$ forces for light nuclei (see Section 4). However, until recently few-body fits have not sufficiently constrained 3N force contributions at higher density such that calculations of symmetric nuclear matter calculations are predictive. One key challenge is the correct reproduction of nuclear saturation. It has long been known that the particle density in the center of atomic nuclei is approximately $n_{s}=0.16 \mathrm{fm}^{-3}$ over a wide range of masses [6], which means that the density in equilibrium approaches a constant value in the thermodynamic limit. Historically, when a quantitative reproduction of empirical saturation properties has been obtained, it was imposed by hand through adjusting phenomenological short-range three-body forces (see e.g., [44, 45]).

In chiral EFT all short-range couplings of the Hamiltonian are fixed in two- and few-body systems and then used to predict properties of many-body systems. In Section 2 (see figure 7) the convergence pattern of such calculations for the equation of state of symmetric nuclear matter was already discussed. For these calculations the short-range 


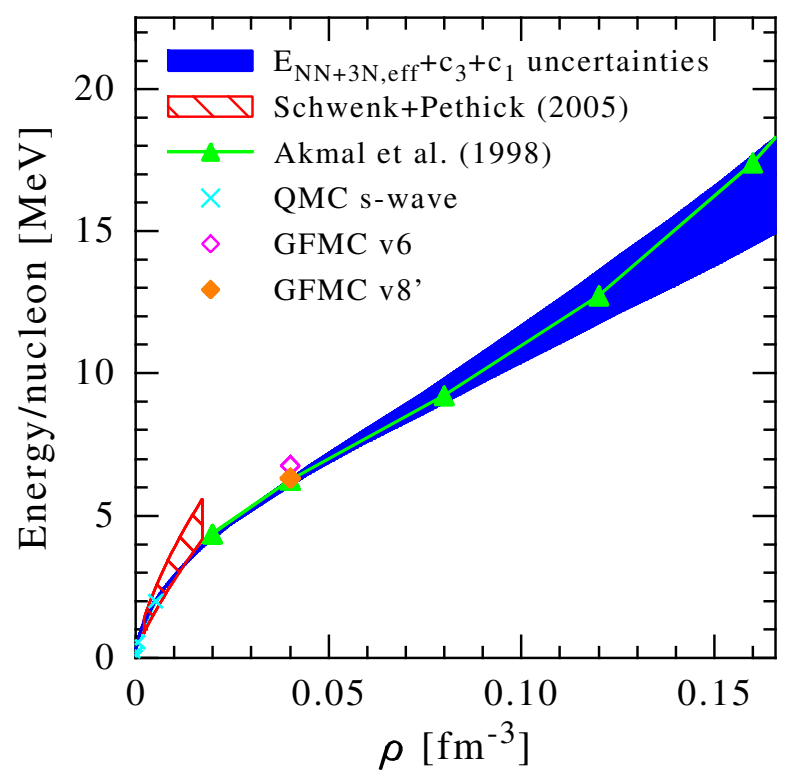

Figure 13. Neutron matter energy as a function of density including NN, 3N and $4 \mathrm{~N}$ forces up to $\mathrm{N}^{3} \mathrm{LO}$ in comparison to other studies (from [35]).

$3 \mathrm{~N}$ forces have been fitted to the experimental values of the ${ }^{3} \mathrm{H}$ binding energy and the radius of ${ }^{4} \mathrm{He}$. The Hartree-Fock results show that nuclear matter is bound even at the simplest level in the many-body expansion. It is encouraging that the results agree with the empirical saturation point, indicated by the grey square, within the uncertainty in the many-body calculation and omitted higher-order many-body forces implied by the cutoff variation. We stress that the cutoff dependence of order $3 \mathrm{MeV}$ around saturation density is small compared to the total size of the kinetic energy $(\approx 23 \mathrm{MeV})$ and potential energy $(\approx-44 \mathrm{MeV})$ at this density. Moreover, the cutoff dependence is smaller at $k_{F}=1.1 \mathrm{fm}^{-1}$, which is the typical density in the interior of medium-mass to heavy nuclei. For all cases in the right panel of figure 7 the nuclear compressibility $K=175-210 \mathrm{MeV}$ is also in the empirical range.

The role of $3 \mathrm{~N}$ forces for saturation is demonstrated in figure 12 . The two pairs of curves show the differences between the nuclear matter results for NN-only and NN plus $3 \mathrm{~N}$ interactions. It is evident that saturation is driven by $3 \mathrm{~N}$ forces. Even for $\Lambda=2.8$ $\mathrm{fm}^{-1}$, which is similar to the lower cutoffs in chiral EFT potentials, symmetric nuclear matter does not even saturate in the plotted density range. While $3 \mathrm{~N}$ forces drive saturation for low-momentum interactions, the $3 \mathrm{~N}$ contributions are not unnaturally large (see also figure 14 and discussion below).

\subsection{Neutron matter}

Neutron matter is a particularly useful testing ground for chiral forces because only the long-range $2 \pi$-exchange $3 \mathrm{~N}$ forces contribute [35], which implies that all three- and four-neutron $(4 \mathrm{~N})$ forces are predicted up to $\mathrm{N}^{3} \mathrm{LO}$. In addition, as a result of weaker 

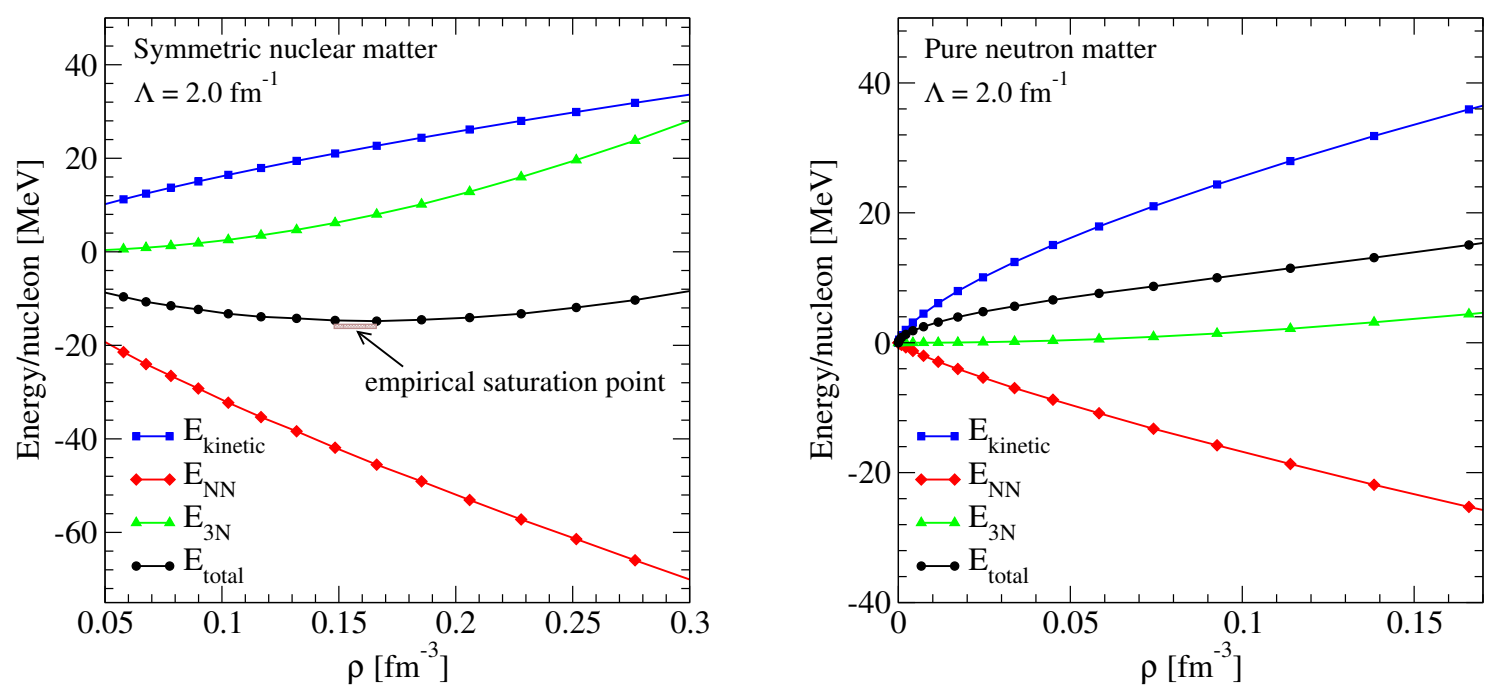

Figure 14. Hierarchy of many-body contributions as function of density for symmetric nuclear matter [23] and pure neutron matter [35. $E_{\mathrm{NN}}$ denotes the energy contributions from $\mathrm{NN}$ interactions and $E_{3 \mathrm{~N}}$ all contributions which include at least one $3 \mathrm{~N}$ interaction.

tensor forces between neutrons and the absence of short-range $3 \mathrm{~N}$ forces, neutron matter behaves more perturbatively than symmetric nuclear matter. This results in very small theoretical uncertainties of the neutron matter calculations, mainly due to uncertainties of the low-energy couplings in chiral EFT [28, 35, 29]. In figure 13 we present the neutron matter energy including the uncertainties of the low-energy constants, indicated by the blue band, in comparison to other approaches. These include Greens Function Monte Carlo (GFMC) [46], Quantum Monte Carlo (QMC) [47, results of Akmal et al. [44, and difermion EFT results for lower densities [48]. The results derived from chiral EFT interactions correspond to a nuclear symmetry energy of $S_{v}=30.4-33.6$ $\mathrm{MeV}$. Compared to the empirical range $S_{v}=25-35 \mathrm{MeV}$ [49], the microscopic range of $\approx 3 \mathrm{MeV}$ is very useful and comparison to experiment could also provide guidance for improved constraints on the values of the low-energy constants.

The size of the separate contributions from the kinetic energy, NN forces, and $3 \mathrm{~N}$ forces for symmetric nuclear matter and neutron matter as a function of density for a RG cutoff of $\Lambda=2.0 \mathrm{fm}^{-1}$ are shown in figure 14 . The empirical saturation point for symmetric nuclear matter is again indicated by the rectangle in the left panel. Here it is obvious that the separate contributions are much larger than the sum of all terms (black line) and that saturation is the result of a delicate interplay of these terms. Furthermore, the contributions from $3 \mathrm{~N}$ forces grow faster with density than those from $\mathrm{NN}$ forces due to the additional nucleon involved. This implies that beyond some critical density region the chiral hierarchy of many-body forces will break down and Hamiltonians based on chiral EFT will not be useful anymore. However, for symmetric nuclear matter we still find that the contributions involving $3 \mathrm{~N}$ forces are about a factor 3 smaller than those from NN forces around twice nuclear saturation density. For neutron matter the 


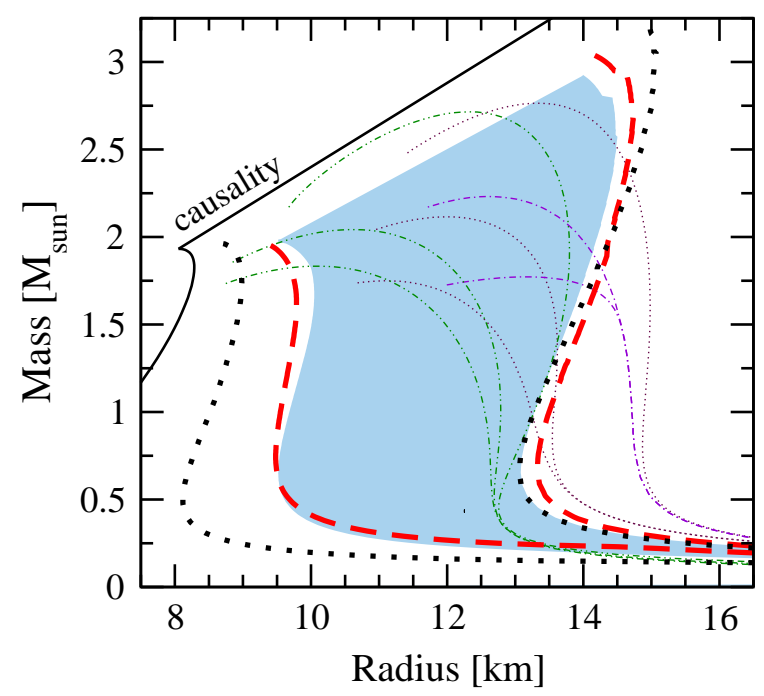

Figure 15. Neutron star mass-radius results for the equations of state based for $M>$ $1.97 M_{\odot}$. The blue band shows the radius constraint based on RG-evolved interactions plus $3 \mathrm{~N}$ interactions based on chiral EFT. The dashed and dotted lines represent a selection of other EOSs which are currently used in astrophysical simulations and neutron star calculations. See Ref. [50] for details.

$3 \mathrm{~N}$ forces play a less significant role and give only moderate repulsive contributions.

\subsection{Applications to neutron stars}

The neutron matter results have direct implications for the properties of neutron stars. Since core densities inside a neutron star can reach several times the interior density of heavy nuclei, much higher than the maximal density up to which the equation of state can be calculated reliably, it is necessary to extend the microscopic results to higher densities. This can be achieved by employing a general strategy that does not rely on assumptions about the nature of the nuclear constituents and their interactions at high densities: by choosing a piecewise polytropic ansatz [51] and limiting the range of the free parameters by physics and constraints from neutron star observations [52. In particular, the following two constraints have been used: (a) the speed of sound remains smaller than the speed of light for all densities, and (b) the EOS is able to support a neutron star of mass $M \geq M_{\min }=1.97 M_{\odot}$, which is currently the heaviest confirmed observed neutron star mass [53]. This results in uncertainty bands for the equation of state and for neutron star radii. The constraints for neutron stars are shown in figure 15. For a typical neutron star of mass $M=1.4 M_{\odot}$ a radius range $R=10.0-13.7 \mathrm{~km}$ is found. For comparison, a selected set of alternative EOSs are shown that are currently used in astrophysical simulations (for details see Ref. [54]). It is evident that many of these are inconsistent with constraints derived from interactions based on chiral EFT. 

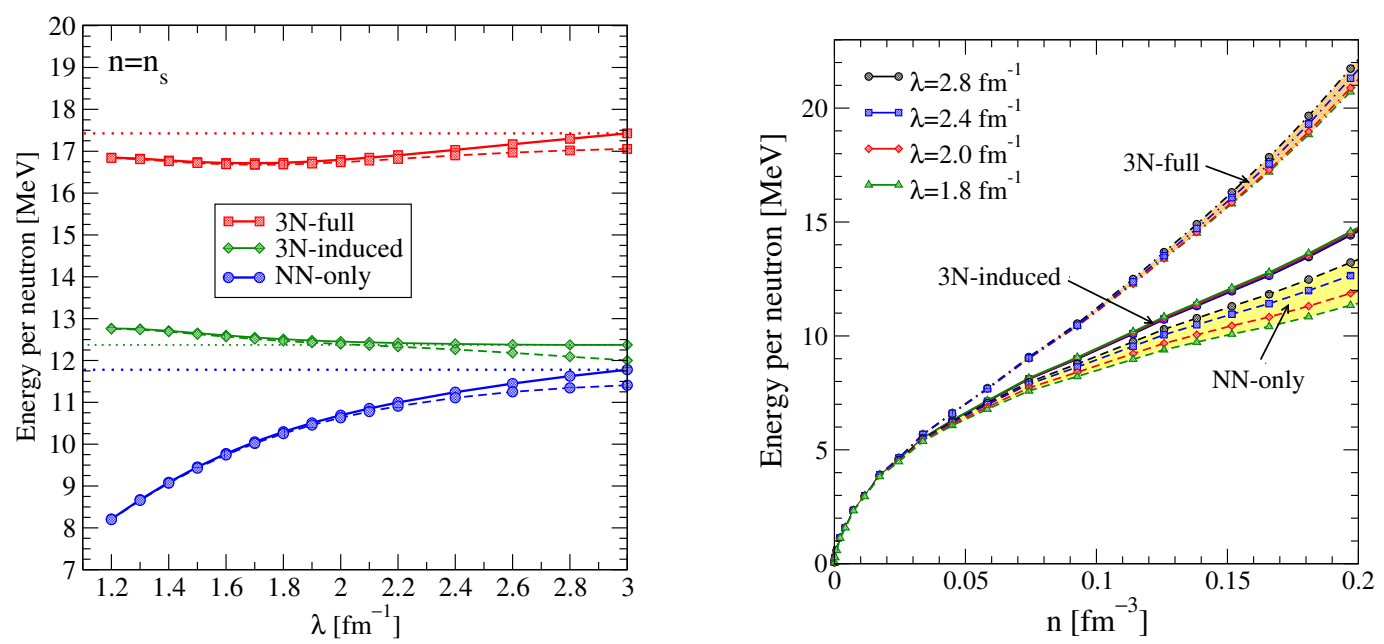

Figure 16. Neutron matter equation of state based on consistently-evolved NN+3N interactions. See Ref. [55] for details.

\subsection{First results based on consistently evolved three-nucleon forces}

All results for neutron and symmetric nuclear matter presented so far were based on evolved NN interactions plus $3 \mathrm{~N}$ interactions that were fixed at the low-momentum scale rather than the chiral EFT cutoff scale (strategy (b) in section 2). Thanks to recent developments it is now also possible to consistently evolve $3 \mathrm{~N}$ interactions [36], which can be used directly in microscopic calculations of infinite nuclear matter (strategy (c) in section 2). In figure 16 we present very recent results for neutron matter based on interactions derived in this framework [55]. For these calculations the $3 \mathrm{~N}$ contributions to the equation of state have been calculated in Hartree-Fock approximation. This approximation is expected to be reliable at small $\lambda$, whereas at larger scale higherorder contributions are known to be important [35]. The left panel shows results for the energy per neutron at saturation density as a function of the SRG resolution scale $\lambda$ using the three approximations "NN-only", "3N-induced" and "3N-full" (see also figure 9). Evidently, neglecting all $3 \mathrm{~N}$ interactions in the $\mathrm{RG}$ evolution results in a significant resolution-scale dependence with a energy variation of about $3.5 \mathrm{MeV}$. By including the induced contributions the variation is significantly reduced to about 400 $\mathrm{keV}$, with the major part of this variation happening at small $\lambda$. By including also initial $3 \mathrm{~N}$ interactions, a total variation of about $600 \mathrm{keV}$ is found. The yellow band indicates the size of the second-order contributions at $\lambda=\infty$, which can be calculated using the framework from [35]. The fact that the observed variation of the energy is within this band suggests that the results can be systematically improved by including higher-order contributions in the many-body expansion. This work is currently in progress.

In the future it will also be possible to systematically study response functions of nuclear matter within this framework. For this it will be essential to also consistently evolve other operators (see Section 5). 


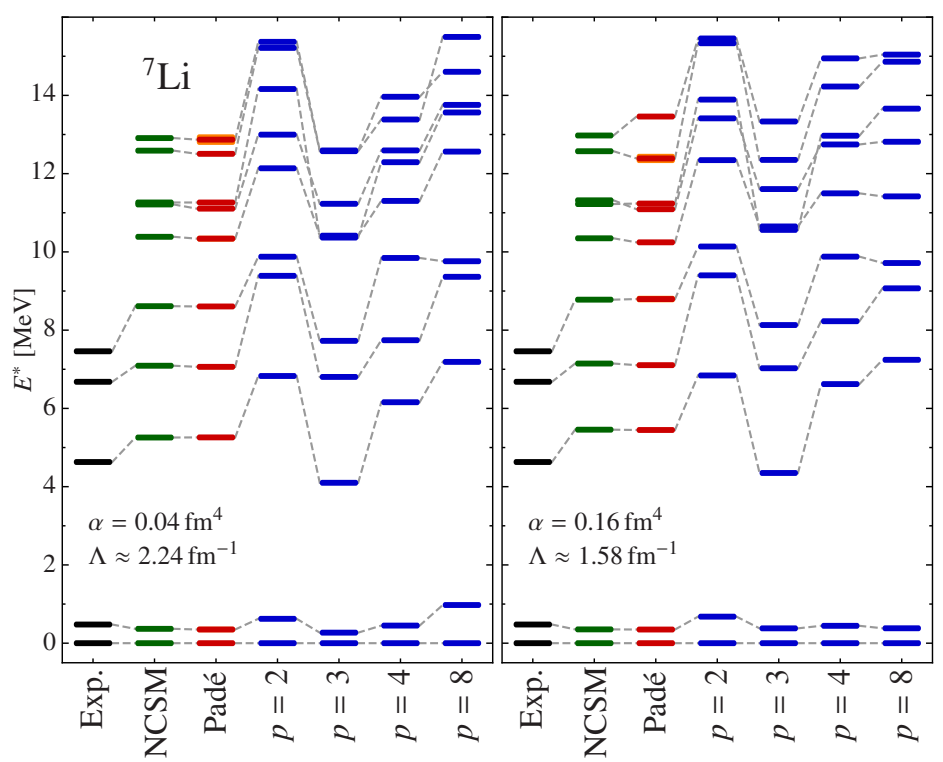

Figure 17. Excitation energies in ${ }^{7} \mathrm{Li}$ calculated in degenerate Rayleigh-Schrödinger MBPT at order $p=2,3,4$, and 8 for a $\mathrm{N}^{3} \mathrm{LO}$ two-body interaction evolved using the $\mathrm{SRG}$ to two different resolutions ( $\Lambda$ here is the same as $\lambda$ used elsewhere) compared to experiment, exact NCSM calculations, and the Padé resummed result [57] A fixed harmonic oscillator model space is used for all calculations.

\section{Applications to finite nuclei}

The softening of nuclear forces by RG evolution has made possible many new calculations of finite nuclei with a wide variety of techniques. In this section we present a selection of recent results, highlighting the present successes, future potential, and open problems.

\subsection{Many-body perturbation theory in finite nuclei}

The apparent success of low-order many-body perturbation theory (MBPT) in infinite nuclear matter with low-momentum potentials has been tested for finite nuclei by Roth and collaborators, who have performed calculations in high-order Rayleigh-Schrödinger MBPT using SRG-evolved two-body interactions (based on an initial $\mathrm{N}^{3} \mathrm{LO}$ interaction) for both closed-shell [56] and open-shell [57] nuclei. The calculations for ${ }^{7} \mathrm{Li}$ in degenerate MBPT in a fixed harmonic oscillator model space shown in figure 17 are typical. Even for very soft potentials (e.g., the right panel) the perturbation series diverges. However, a simple resummation with Padé approximants (see Refs. [56, 57] for details) results in stable energies in very good agreement with exact no-core shell-model (NCSM) calculations using the same model space. Future work will include three-body forces and study applications to heavier open-shell nuclei and alternative partitionings of the Hamiltonian (e.g., using a Hartree-Fock unperturbed basis).

The softening of potentials also enables the direct use of perturbative methods 

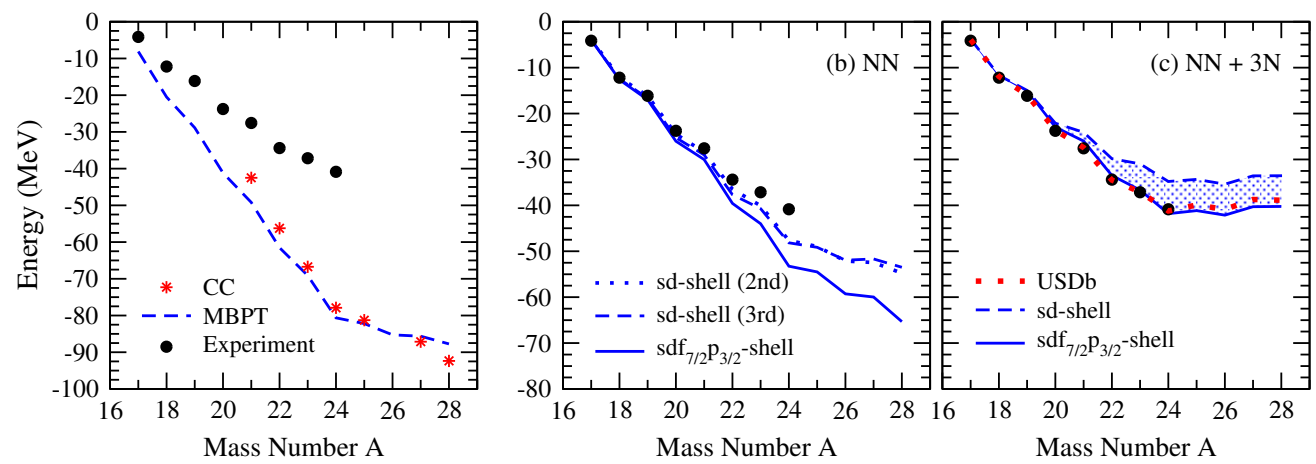

Figure 18. Calculations of oxygen isotope ground-state energies compared to experiment [59]. Left: MBPT compared to coupled cluster theory with the same low-momentum NN-only interaction; middle: MBPT NN-only at 2nd and 3rd order, with empirical SPEs; right: MBPT at 3rd order from NN and $3 \mathrm{~N}$ forces with calculated MBPT SPEs, plus the phenomenological USDb model.

in microscopic valence-shell calculations, in which a small number of nucleons outside a closed-shell core interact via an effective interaction treated in MBPT (with a nonperturbative transformation to remove the energy dependence of the MBPT effective Hamiltonian [58]). One application is to identify the effects of $3 \mathrm{NF}$ on the location of the neutron dripline: the limits of nuclear existence where an added neutron is no longer bound-it "drips" away. This limit is not always easily understood. For example, experiment shows that as neutrons are added to stable ${ }^{16} \mathrm{O}$, the neutrons stay bound until ${ }^{24} \mathrm{O}$. But adding one more proton to get fluorine extends the dripline all the way to ${ }^{31} \mathrm{~F}$. A valence-shell MBPT calculation building on ${ }^{16} \mathrm{O}$ and using an RG-softened NN potential is shown in the left panel of figure 18, where it is validated against nonperturbative coupled-cluster (CC) calculations with the same interaction and consistent single-particle energies (SPEs) [59]. Using empirical SPEs improves the agreement with experimental energies (middle panel), but these microscopic NN-only calculations show too much attraction beyond $A=24$, so that ${ }^{28} \mathrm{O}$ is the predicted dripline. The missing physics from $3 \mathrm{NF}$ is indicated schematically in figure 19; pairs of valence nucleons feel an additional repulsion from long-range $3 \mathrm{NF}$ including a core nucleon [33. When this effect is included, with a chiral $\mathrm{N}^{2} \mathrm{LO} 3 \mathrm{NF}$ and calculated SPEs, the dripline is at ${ }^{24} \mathrm{O}$ [59] (right panel and see Ref. [33] for an earlier calculation with empirical SPEs). The 3NF can also account for how the phenomenological shell model adjusts to reproduce the same trends with $A$ [59].

When applied in the calcium isotopes, this microscopic MBPT method with 3NF predicted that two-neutron separation energies should be significantly larger than found in previous experiments. However, new high-precision measurements using a Penning trap show excellent agreement with the MBPT predictions (see figure 20] 60]. Once again, the theoretical ingredients are SPEs and residual two-body interactions, which are all calculated microscopically from $\mathrm{NN}$ and $3 \mathrm{~N}$ forces [60, 61]. The $\mathrm{NN}$ forces are chiral EFT N ${ }^{3} \mathrm{LO}$ interactions evolved with a smooth $V_{\text {low } k} \mathrm{RG}$ to a low-momentum 
cutoff of $\Lambda=2.0 \mathrm{fm}^{-1}$ to improve the convergence of the MBPT. Other calculations show the predictive power of the method for shell structure and pairing gaps [62], excitation spectra [59], and properties of proton-rich nuclei [61]. On-going work seeks to extend the framework to include continuum effects for weakly bound or unbound states, to develop nonperturbative methods for valence shell interactions 63, to relate to phenomenological models, and to quantify theoretical uncertainties.

\subsection{Ab initio calculations with three-nucleon forces}

The frontier for RG-based ab initio calculations of finite nuclei using microscopic internucleon forces is the inclusion of 3NF. The SRG has made possible the inclusion of consistently evolved $3 \mathrm{NF}$ in a harmonic oscillator basis [27, 30], which means 3NF are present in the initial Hamiltonian but also induced as a result of RG evolution.

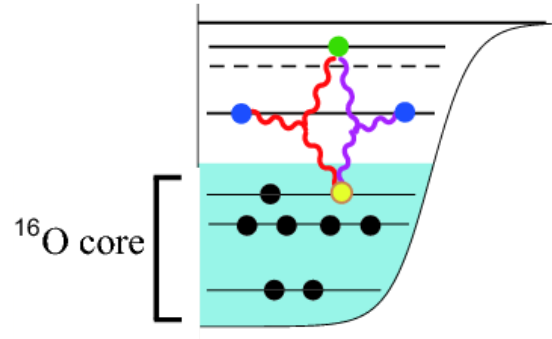

Figure 19. Interaction between valence neutrons and a core nucleon in an oxygen isotope through a three-body force 33 .

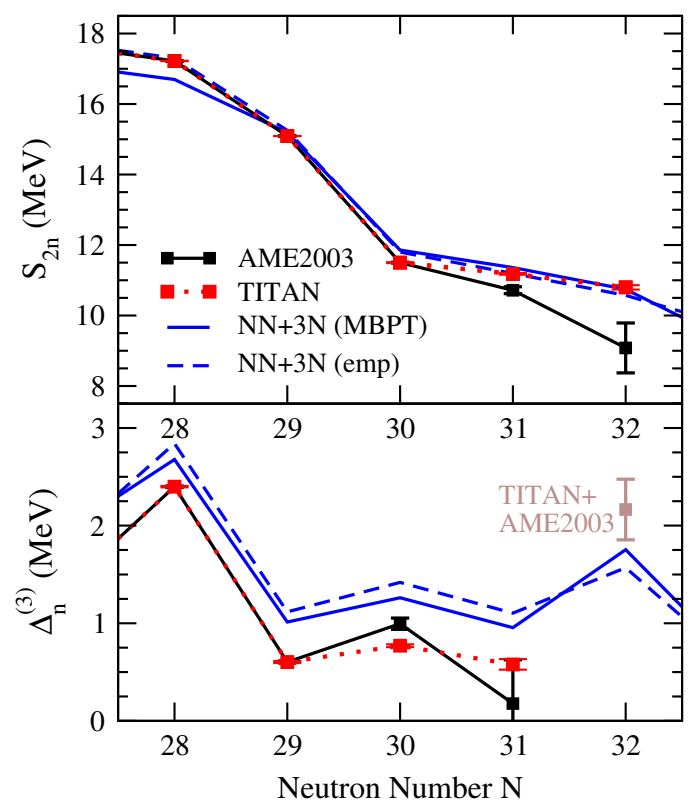

Figure 20. Predictions for two-neutron separation energy and pairing gaps in calcium isotopes including three-body forces compared to new experimental measurements 60]. 
The $\mathrm{NN}+3 \mathrm{~N}$ interaction at lower resolution is found to have the same improved convergence properties for configuration interaction calculations as found earlier for NN-only calculations (e.g., see Refs. [64]). Despite the softening, the factorial growth of basis spaces in the no-core shell model (NCSM) still limits calculations in a complete model space to light nuclei (roughly up to ${ }^{12} \mathrm{C}$ ).

To go to larger nuclei, Roth and collaborators have adapted importance truncation from quantum chemistry to nuclear calculations [65]. This method greatly reduces the size of the Hamiltonian matrix to be diagonalized by identifying the most important matrix elements. While there are still some open questions about uncertainties [66], results are very promising. At the same time, RG-evolved $3 \mathrm{NF}$ has been added to nuclear coupled cluster (CC) calculations [67, 68, 69], first in a normal-ordered approximation [31, 32] and then with the full 3NF interaction [70]. In figure 21. importance truncated no-core shell model (IT-NCSM) results are compared to coupled cluster calculations (at the CCSD level) for ${ }^{16} \mathrm{O}$. In the top panels, an initial NN-only interaction is evolved to four different SRG resolutions, including the induced 3NF [32]. The results are in good agreement and largely independent of the SRG flow parameter.

However, the lower panels in figure 21. show that while good convergence is still found when initial $3 \mathrm{NF}$ are included, the flow is no longer unitary at the $10 \mathrm{MeV}$ level. Detailed investigations [31, 32] show that the long-range 3NF is the source of apparent large $4 \mathrm{NF}$ contributions for oxygen and heavier nuclei, causing a strong dependence on the flow parameter. However, by using a lower cutoff for the initial $3 \mathrm{NF}$, cutoff independence is largely restored and good agreement with experimental binding energies is achieved despite fitting only to few-body properties [31, 32]. This is illustrated for two calcium isotopes in figure 22, with more examples in Refs. [32, 70]. Work is in progress to check whether an alternative SRG 3NF evolution (e.g., in a momentum basis) or the use of alternative SRG generators may be able to better control the RG evolution of the initial 3NF.

\subsection{Ab-initio reactions with $R G$-evolved forces}

One of the principal aims of recent large-scale collaborations in low-energy nuclear physics (e.g., the UNEDF and NUCLEI projects [72, 73, 74]) is to calculate reliable reaction cross sections for astrophysics, nuclear energy, and national security, for which extensions of standard phenomenology are insufficient. The interplay of structure and reactions is essential for a successful description of exotic nuclei as well. A powerful approach to implementing this interplay is the ab initio no-core shell model/resonatinggroup method (NCSM/RGM), which treats bound and scattering states within a unified framework using fundamental interactions between all nucleons [75, 76]. Although only a few years under development, a wide range of applications is already possible. Figure 23 shows the first-ever ab-initio calculation of the ${ }^{7} \mathrm{Be}(p, \gamma)^{8} \mathrm{~B}$ astrophysical S-factor [71], a reaction important for solar neutrino physics. This calculation uses NCSM/RGM with an $\mathrm{N}^{3} \mathrm{LO} \mathrm{NN}$ interaction evolved by the SRG to the special value of $\lambda=1.86 \mathrm{fm}^{-1}$, 


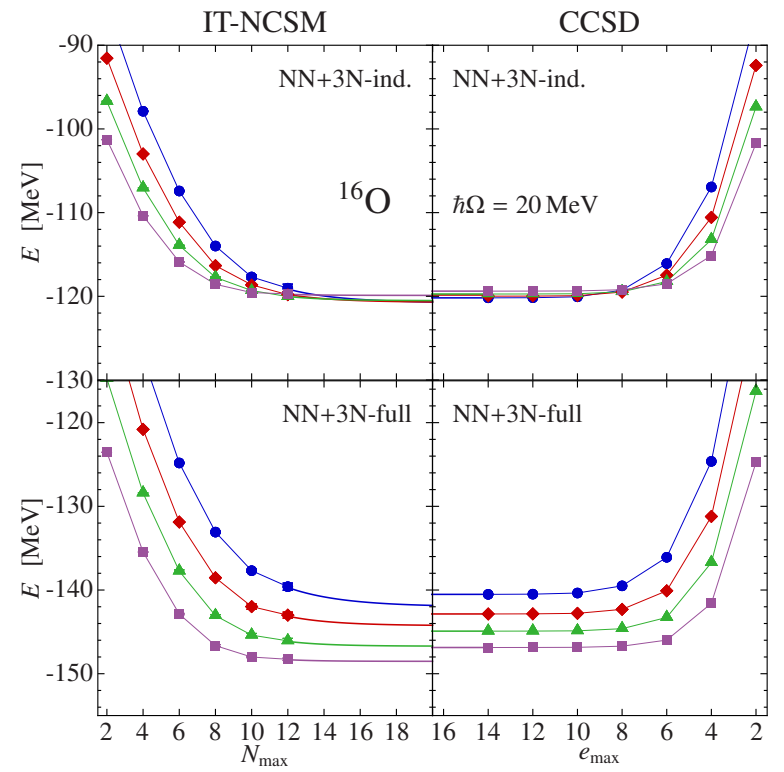

Figure 21. Comparison of IT-NCSM and CC calculations for ${ }^{16} \mathrm{O}$ at four SRG resolutions without (above) and with (below) an initial three-body force (from Ref. [32]).

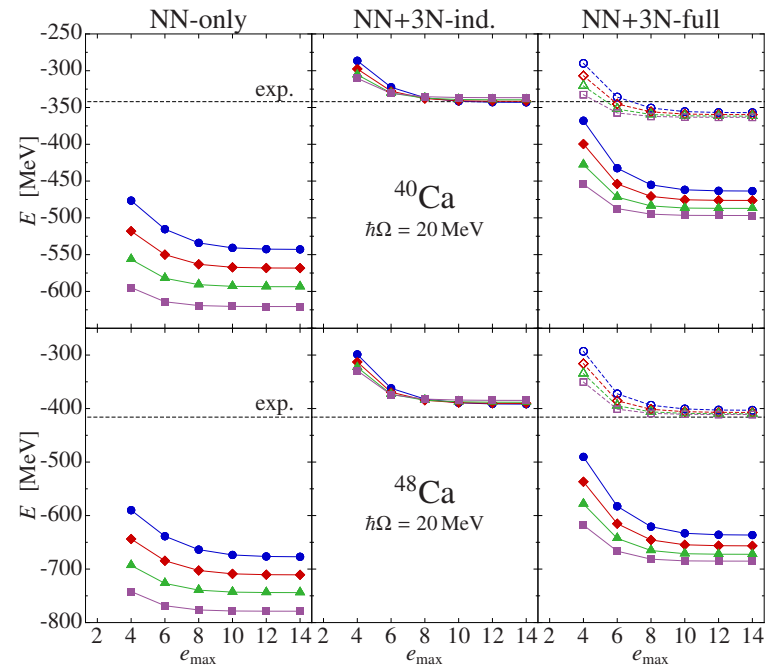

Figure 22. Convergence of CC calculations for ${ }^{40} \mathrm{Ca}$ and ${ }^{48} \mathrm{Ca}$ with only NN (left), $\mathrm{NN}+3 \mathrm{~N}$-induced (middle), and NN+3N-full (right) [32].

chosen to reproduce the observed separation energy for ${ }^{8} \mathrm{~B}$, which is important for an accurate reproduction of the low-energy behavior. Both the normalization and the shape of $S_{17}$ are predicted. Other recent applications include the first ab initio manybody calculations of ${ }^{3} \mathrm{H}(d, p){ }^{4} \mathrm{He}$ and ${ }^{3} \mathrm{H}(d, n){ }^{4} \mathrm{He}$ fusion reactions, which reproduce the experimental $Q$-value of both within 1\% [77]. The convergence of the latter calculations is shown in figure 24; the ability to tune the SRG interaction is again used to compensate for omitted higher-order effects, such as the 3NF. Calculations including consistent SRG- 


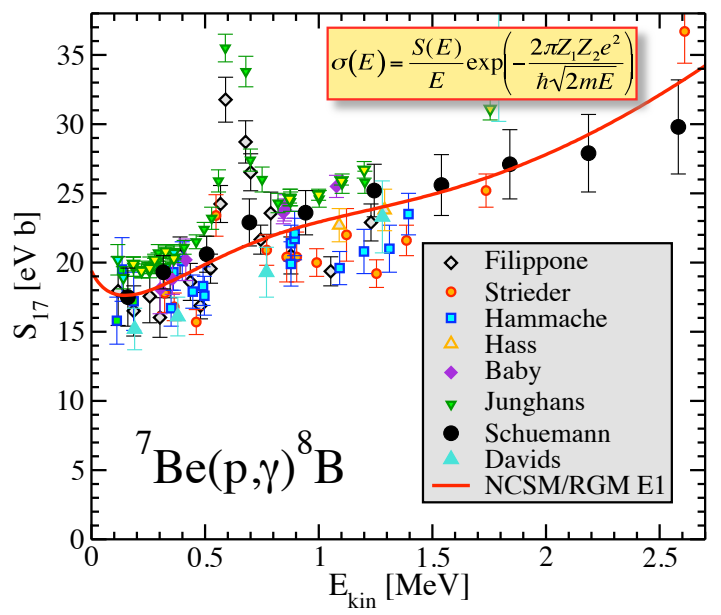

Figure 23. First ever ab initio calculations of ${ }^{7} \mathrm{Be}(p, \gamma)^{8} \mathrm{~B}$ astrophysical S-factor [71]. Uses SRG-N3LO with $\lambda=1.86 \mathrm{fm}^{-1}$.

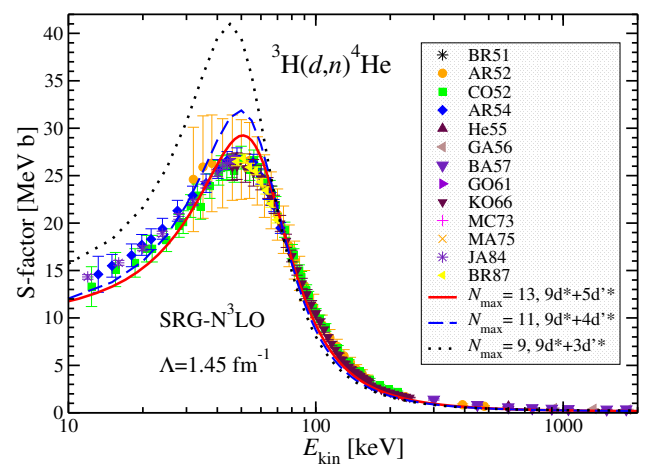

Figure 24. Calculated ${ }^{3} \mathrm{H}(d, n){ }^{4} \mathrm{He}$ S-factor at three model space sizes compared to experimental data for an SRG- ${ }^{3} \mathrm{LO} N \mathrm{~N}$ potential with $\lambda=1.45 \mathrm{fm}^{-1}$ tuned to compensate for missing higher-order effects [77].

evolved $3 \mathrm{NF}$ will be available in the near future [78]. Another related new development is the no-core shell model with continuum (NCSMC), which is a unified approach to nuclear bound and continuum states [79]. A recent proof-of-principle NCSMC calculation uses a realistic soft SRG-N ${ }^{3} \mathrm{LO}$ nucleon-nucleon potential to describe resonances in ${ }^{7} \mathrm{He}$ [79].

An alternative approach to nuclear reactions of light nuclei is the fermionic molecular dynamics (FMD) method, which uses anti-symmetrized many-body states built from localized (Gaussian) single-particle wave packets to provide a fully microscopic calculation with both bound and scattering states described consistently. The effective interaction is derived by the Unitary Correlation Operator Method (UCOM) [80], which eliminates short-range and tensor correlations by unitary transformations guided by the SRG. An example of the capabilities of FMD is shown in figure 25, where the calculated astrophysical S-factor for ${ }^{3} \mathrm{He}(\alpha, \gamma){ }^{7} \mathrm{Be}$ is compared to new high-quality experimental results [81, 82, 83]. FMD is the only model that has been able to describe both the energy dependence and normalization of the new data. 


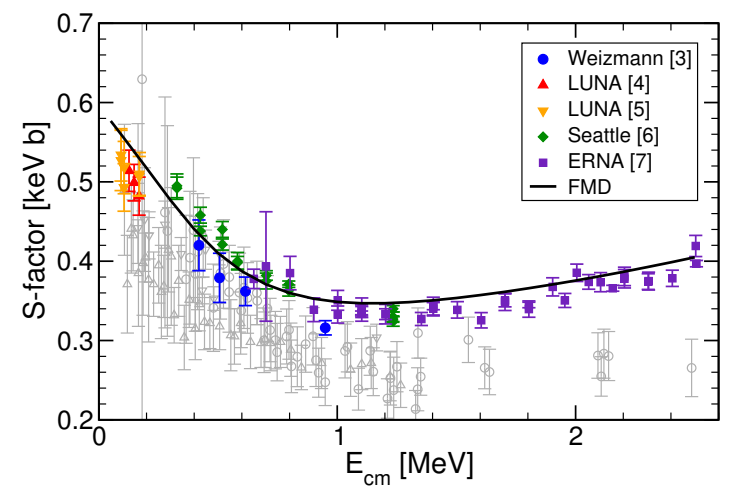

Figure 25. Astrophysical S-factor for ${ }^{3} \mathrm{He}(\alpha, \gamma){ }^{7} \mathrm{Be}$ calculated using FMD [81, 82, 83,

\subsection{In-medium Similarity Renormalization Group}

The in-medium SRG (IM-SRG) for nuclei, developed recently by Tsukiyama, Bogner, and Schwenk [84], applies the RG flow equations in an $A$-body system using a different reference state than the vacuum. The key to the IM-SRG is the use of normal-ordering with respect to the finite-density reference state. That is, starting from the secondquantized Hamiltonian with two- and three-body interactions,

$$
\begin{aligned}
H & =\sum_{12} T_{12} a_{1}^{\dagger} a_{2}+\frac{1}{(2 !)^{2}} \sum_{1234}\langle 12|V| 34\rangle a_{1}^{\dagger} a_{2}^{\dagger} a_{4} a_{3} \\
& +\frac{1}{(3 !)^{2}} \sum_{123456}\left\langle 123\left|V^{(3)}\right| 456\right\rangle a_{1}^{\dagger} a_{2}^{\dagger} a_{3}^{\dagger} a_{6} a_{5} a_{4},
\end{aligned}
$$

all operators are normal-ordered with respect to a finite-density Fermi vacuum $|\Phi\rangle$ (for example, the Hartree-Fock ground state or the non-interacting Fermi sea in nuclear matter), as opposed to the zero-particle vacuum. Wick's theorem can then be used to rewrite $H$ in normal-ordered form, which reshuffles the contributions. For example, the zero-, one-, and two-body normal-ordered terms will now have contributions from the original three-body term in Eq. (9), which are in practice the dominant pieces. Therefore, truncating the in-medium SRG equations to two-body normalordered operators will (to good approximation) evolve induced three- and higher-body interactions through the density-dependent coefficients of the zero-, one-, and two-body operators. The appealing consequence is that, unlike the free-space SRG evolution, the in-medium SRG can approximately evolve $3, \ldots, A$-body operators using only twobody (or three-body) machinery. However, also in contrast to the free-space SRG, the in-medium evolution must be repeated for each nucleus or density.

The IM-SRG decouples the ground state of the many-body Hamiltonian from all excitations (see figure 26) by means of a continuous unitary transformation, which is characterized by a suitable choice of dynamical generator. The method is implemented by solving a set of flow equations analogous to the free-space SRG approach. In principle, the IM-SRG is an exact method, but in practice a hierarchy of truncations is needed to close the set of equations; this hierarchy allows systematic improvements of the method. 


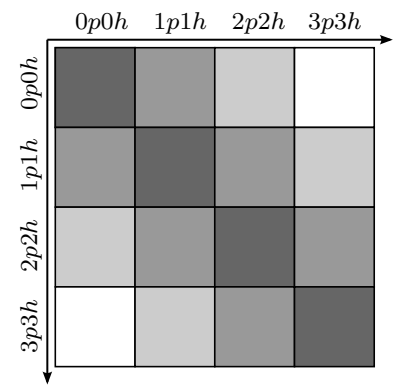

$\langle i|H(0)| j\rangle$

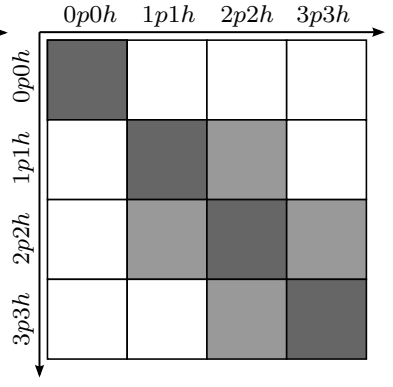

$\langle i|H(\infty)| j\rangle$

Figure 26. Schematic representation of the initial and final Hamiltonians in the manybody Hilbert space spanned by particle-hole excitations of the reference state [85].

For practical applications, the IM-SRG has two appealing features. First, most of the effects of higher-order induced many-body forces are automatically included (unlike freespace SRG evolution). Second, it exhibits polynomial scaling with the basis size, which allows us to perform calculations for nuclei that are not accessible by other ab initio many-body methods such as the NCSM or GFMC.

While the IM-SRG equations are of second order in the interactions, the flow equations build up non-perturbative physics through the evolution. In terms of diagrams, one can imagine iterating the SRG equations in increments of the flow parameter $\delta s$. At each additional increment $\delta s$, the interactions from the previous step are inserted back into the right side of the SRG equations. Iterating this procedure, one sees that the SRG accumulates complicated particle-particle and particle-hole correlations to all orders (see figure 2 in [85]). With an appropriate choice of generator, the Hamiltonian is driven towards the diagonal, as indicated schematically in figure 26. This means that Hartree-Fock becomes increasingly dominant with the off-diagonal matrix elements being driven to zero [86].

The in-medium SRG is well suited as an ab initio method for finite nuclei. Figure 27 shows the rapid convergence of energy calculations. Results for closed shell nuclei up to ${ }^{56} \mathrm{Ni}$ are shown in figure 28, which shows a striking improvement in the isotopic trends with the inclusion of initial 3NF. The IM-SRG truncated at the normal-ordered twobody level gives results comparable to IT-NCSM and to coupled-cluster calculations with some triples corrections ( $\Lambda$-CCSD $(T)$, see Ref. [85]).

To allow systematic investigations of trends in ground-state energies (and other observables along complete isotopic chains), Hergert and collaborators have generalized the IM-SRG to the Multi-Reference IM-SRG (MR-IM-SRG) [87]. This approach allows the calculations to be extended to several hundred known spherical open-shell nuclei while retaining a polynomial numerical scaling. Figure 29 shows a first application to the oxygen chain [87, which is validated against CC and IT-NCSM with the same Hamiltonians. The inclusion of an initial $3 N$ Hamiltonian is seen to be needed to obtain agreement with experimental data.

Here are some in-progress extensions of the IM-SRG and MR-IM-SRG: 

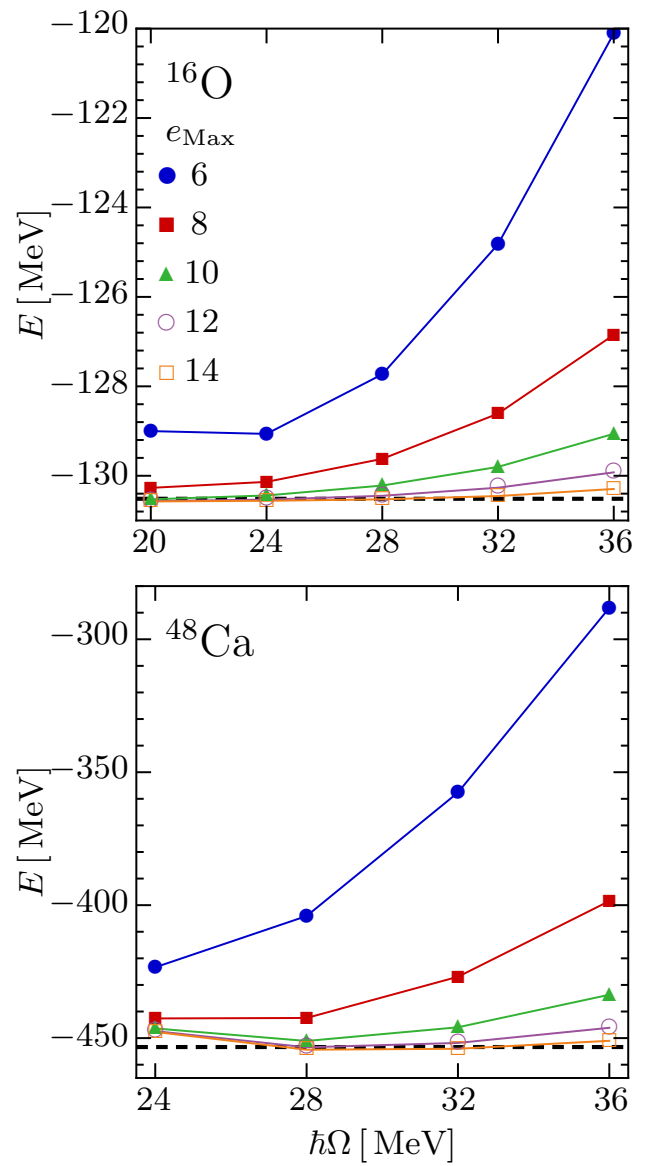

Figure 27. Examples of the convergence of the IM-SRG ground-state energy for different harmonic oscillator basis frequencies $\hbar \Omega$ and different basis sizes $e_{\text {Max }}$ for an initial $\mathrm{NN}+3 \mathrm{~N}$ Hamiltonian, which is first evolved in free space to $\lambda=2.0 \mathrm{fm}^{-1}$. It is evident that the results become independent of the oscillator frequency with increasing basis size. See Ref. [85] for details.

- The calculation of excited states. There are several possibilities to achieve this, for instance, a modification of the currently used generator to decouple multiple states rather than just the ground state in the Hamiltonian's spectrum, or the adaptation of equations-of-motion methods like in coupled cluster (see e.g., [89]). This would ultimately provide the capability to calculate transition densities, which can be used as input by the nuclear reaction community.

- As in the free-space SRG, all observables besides the Hamiltonian have to be evolved consistently, which can be implemented by evolving the creation and annihilation operators in which the flowing operators are represented rather than the matrix elements of these operators. The transformed basis operators, or alternatively transformed many-body density matrices, could then be used to calculate expectation values for any observable of interest in an economic fashion.

- With a modified generator to allow for the decoupling of a pre-defined valence space, an evolved Hamiltonian obtained from a closed-shell IM-SRG calculation 


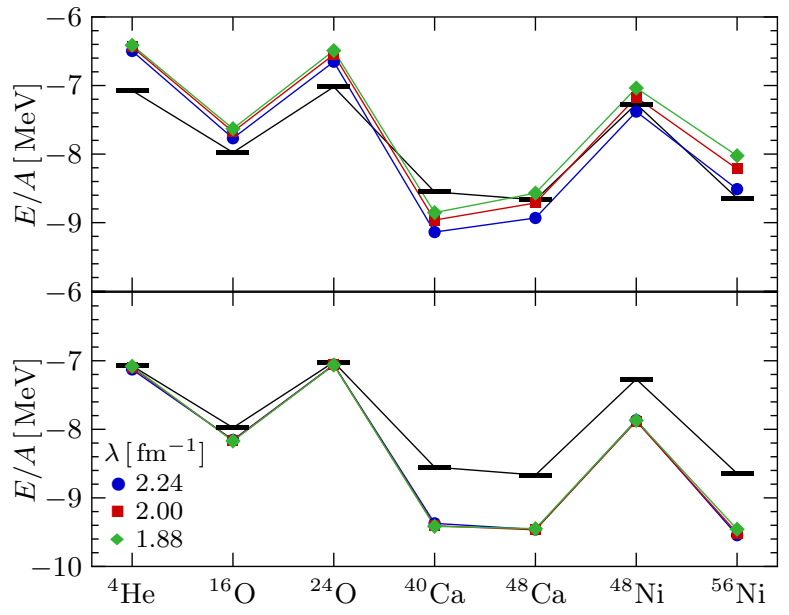

Figure 28. Ground-state energies per nucleon of closed-shell nuclei using IM$\mathrm{SRG}(2)$ at different SRG resolution scales $\lambda$ [85]. Results with $\mathrm{NN}+3 \mathrm{~N}$-induced Hamiltonians are shown on top while the bottom includes initial 3NF. The black bars are experimental energies.

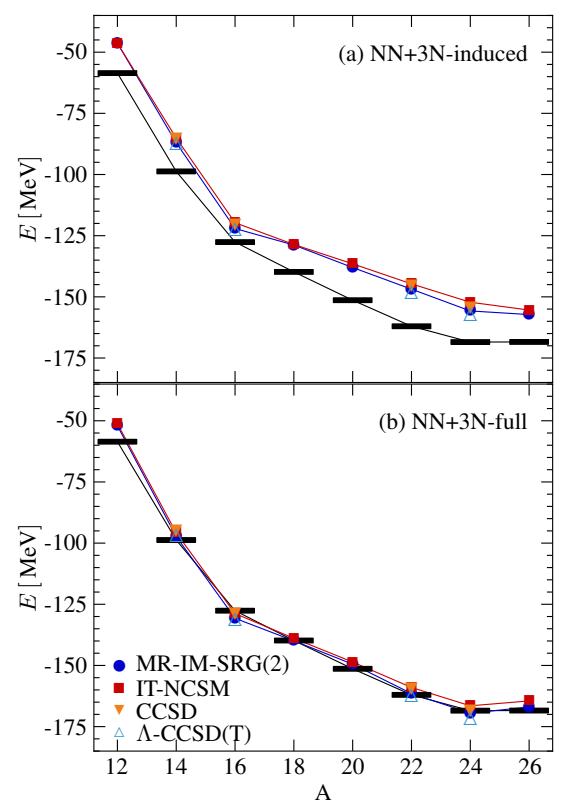

Figure 29. Ground-state energy of oxygen isotopes from the IM-SRG for different SRG parameters $\lambda$ [87. Top: Chiral NN Hamiltonian and induced 3N interaction (no initial 3N terms). Bottom: Consistently evolved chiral NN and 3N Hamiltonian. The pluses are experimental data from Ref. [88].

can be used as a microscopic input to traditional shell model approaches [63]. Shell Model calculations with IM-SRG Hamiltonians will yield complete spectroscopic information, and are in this sense complementary to the direct calculation of excited states in the IM-SRG framework. A combined IM-SRG/Shell Model approach is the most practical way to study deformed nuclei in the near future. 
- IM-SRG is being adapted for nuclear matter by implementing it in a periodic box. This will provide a non-perturbative assessment of infinite matter calculations.

\subsection{Other applications and future directions}

We have only described a fraction of the recent and on-going work on finite nuclei that exploits RG methods. However, here we briefly describe some of the other important developments that make use of low-momentum interactions (see also Refs. [90, 91]).

- In Ref. [92, the binding energy and radii of the two-neutron halo nucleus ${ }^{6} \mathrm{He}$ have been studied in the hyperspherical harmonics approach based on low-momentum NN interactions. The RG evolution has been found to be essential to obtain converged results of the extended matter radius and of the point-proton radius.

- In Ref. [93], the No-Core Gamow Shell Model (NCGSM), which treats bound, resonant, and scattering states equally, was first applied to study some well-bound and unbound states of the helium isotopes 93 . The density matrix renormalization group (DMRG) method [94] was used to solve the many-body Schrödinger equation. The $V_{\text {low } k}$ RG was used to decouple high from low momentum to improve the convergence of the calculations. RG-evolved low-momentum interactions are now a standard first choice for proof-of-principle or benchmark calculations using new techniques.

- Time-dependent coupled-cluster theory has recently been studied in the framework of nuclear physics [95]. Besides using a low-momentum SRG two-body interaction for their proof-of-principle computations, the authors are able to relate the real and imaginary time evolution of the Hamiltonian to SRG transformations.

- Microscopic calculations of pairing properties in mid- and heavy-mass nuclei are being pursued using the ab-initio self-consistent Gorkov Green's function (SCGGF) framework based on low-momentum interactions [96, 97]. Because in practice a tractable truncation scheme must be implemented, RG-softened interactions are a key ingredient to make finite-order schemes qualitatively and quantitatively usable. Recent results using $3 \mathrm{NF}$ show great promise in reproducing the physics of neutron driplines and truly open-shell systems, increasing the number of medium-mass nuclei accessible by ab initio methods from a few tens to a few hundreds [98, 99, 100].

- Recent work has critically examined the infrared and ultraviolet cutoffs imposed on few- and many-body systems by the use of harmonic oscillator basis expansions, which are common in nuclear physics [101, 102]. While progress toward a theoretically founded understanding of universal infrared extrapolations has been made [103], the ultraviolet situation is less clear. The SRG offers a tool for studying ultraviolet extrapolations, including suggestive but as yet unexplained scaling with the SRG flow parameter [102].

- Quantum Monte Carlo (QMC) methods such as Green's function or auxiliary field diffusion Monte Carlo are powerful methods for nuclear structure calculations but 
have been restricted for technical reasons to local interactions. This has precluded their application with low-momentum interactions. Recent progress has been made toward relaxing this restriction, which has also demonstrated universal behavior of propagators at large imaginary times [104].

- Spectral distribution theory (SDT) has been applied to SRG-evolved Hamiltonians to study the nature of three-body SRG-induced interactions at an operator level [105]. This approach reveals that the SRG-renormalized interaction is essentially two-body driven, with the two-body part extractable in the SDT framework.

- The role of long-distance symmetries within the context of SRG evolution has been explored recently [106]. A particular SRG resolution scale is identified for which the Wigner SU(4) symmetry is almost perfectly realized at the two-body level. This motivates a search for similar symmetry patterns for many-body forces.

- The variable cutoff (or decoupling scale) implemented in RG methods is a useful tool for analyzing scheme-dependent observables in nuclear structure[107]. Recent applications have been made to ab initio spectroscopic factors [108] and to effective single-particle energies (ESPEs) [109]. In the latter case, varying the $V_{\text {low } k}$ cutoff clearly identifies the scale dependence of ESPEs and sets the stage for a future quantitative analysis, which will require the treatment of 3NF [109].

We anticipate many new results from these and other RG-motivated investigations in the near future.

\section{Correlations in nuclear systems}

\subsection{Evolution of operators as an $R G$ frontier}

So far we have focused on the evolution of Hamiltonians, but an RG transformation will also modify the operators associated with measurable quantities. If we do not evolve the operator, then its matrix elements calculated with the wave functions of the flowing Hamiltonian will change. Consider, for example, the expectation of a quadrupole operator in the deuteron. It is naturally defined in coordinate space [110]:

$$
\left\langle Q_{d}\right\rangle=\frac{1}{20} \int_{0}^{\infty} d r r^{2} w(r)(\sqrt{8} u(r)-w(r)),
$$

where $u$ and $w$ are the ${ }^{3} \mathrm{~S}_{1}$ and ${ }^{3} \mathrm{D}_{1}$ deuteron radial wave functions. This expectation value as a function of SRG $\lambda$ is shown in figure 30 . Because the quadrupole operator acts on long distance scales (that is, it predominantly samples the large $r$ part of the relative wave function), the variation with $\lambda$ is relatively small but increases more rapidly at the lowest resolutions. The result is never equal to the electromagnetic quadrupole moment extracted from experiment because the operator in Eq. (10) is only the leading-order piece of the full operator that corresponds to this particular experimental quantity. For each Hamiltonian (either initial or at each evolved resolution), there will be a different consistent operator. 


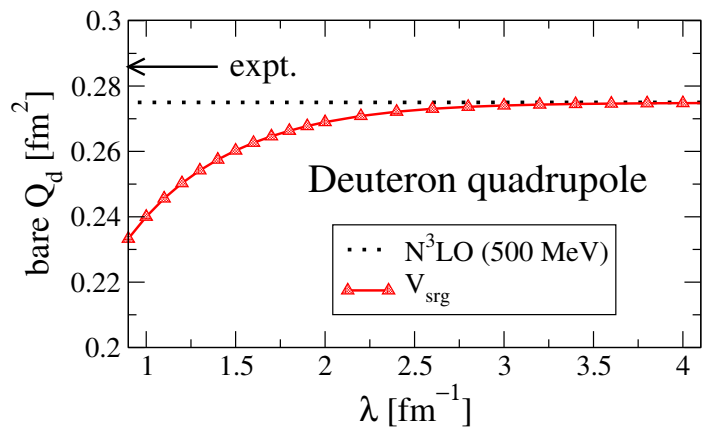

Figure 30. Expectation value in the deuteron of the unevolved quadrupole operator as a function of the SRG resolution $\lambda$ [111. The initial potential is the $\mathrm{N}^{3} \mathrm{LO}(500 \mathrm{MeV})$ chiral NN interaction from Ref. [20]. The experimental value of the electromagnetic quadrupole moment is marked with an arrow.

To enable a general description of experiments, it is essential to be able to start with full operators consistent with the initial Hamiltonian and then to evolve them maintaining this consistency. By working within an EFT framework, we can ensure consistent initial operators because the EFT provides a complete operator basis organized hierarchically by power counting. The second step can be technically difficult, especially because we will inevitably induce many-body operators as we evolve. However, the technology recently developed for the SRG to evolve many-body Hamiltonians (Section 2) can be adapted to evolve other operators at the same time.

The SRG evolution with $s$ (recall $s=1 / \lambda^{4}$ ) of any operator $O$ is given by:

$$
O_{s}=U_{s} O U_{s}^{\dagger}
$$

so $O_{s}$ evolves via

$$
\frac{d O_{s}}{d s}=\left[\left[G_{s}, H_{s}\right], O_{s}\right]
$$

where we must use the same $G_{s}$ to evolve the Hamiltonian and all other operators. While we can directly evolve any operator like this in parallel to the evolution of the Hamiltonian, in practice it is more efficient and numerically robust to either evolve the unitary transformation $U_{s}$ itself:

$$
\frac{d U_{s}}{d s}=\eta_{s} U_{s}=\left[G_{s}, H_{s}\right] U_{s}
$$

with initial value $U_{s=0}=1$, or calculate it directly from the eigenvectors of $H_{s=0}$ and $H_{s}$ :

$$
U_{s}=\sum_{i}\left|\psi_{i}(s)\right\rangle\left\langle\psi_{i}(0)\right|
$$

Then any operator is directly evolved to the desired $s$ by applying Eq. (11) as a matrix multiplication. The second method works well in practice. As with the Hamiltonian, the two-body part of an operator is completely determined by evolution in the $A=2$ space, the three-body part by evolution in the $A=3$ space, and so on. 


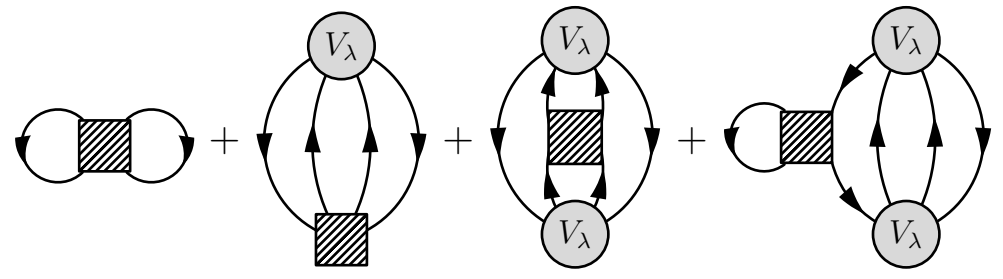

Figure 31. Diagrammatic contributions to the expectation value of a two-body operator (denoted by the shaded square) for the first three orders in MBPT with an NN-only potential.

One option to evaluate operator matrix elements is to apply many-body perturbation theory (MBPT). For example, the diagrams for the first three orders in MBPT of a two-body operator (assumed to depend only on relative momenta) are shown in figure 31 assuming the Hamiltonian has only two-body interactions. In effect, the operator is inserted into the MBPT expansion for the energy in all possible ways (including proper symmetry factors). Diagrammatic perturbation theory of this sort was recently used for an effective double-beta-decay operator by Holt and Engel [112]. The challenge going forward is to include three-body interactions (see figure 11) and the induced three-body parts of SRG-evolved operators.

For finite nuclei beyond the deuteron, the process of evolving and applying operators has several complications. Imagine we start with a one-body operator that we wish to evaluate in an $A$-particle nucleus. As we evolve the operator, first in a 2-particle basis, then a 3-particle basis, and so on (until the desired level of truncation), $n$-body components will be induced and must be kept if matrix elements are to be invariant. These components must be separated because they are be embedded in larger nuclei with different counting factors [113. In addition, we need in general to apply appropriate boosts to the operators before embedding. A flowchart summarizing the procedure is given as figure 49 in Ref. [9]. Proof-of-principle calculations for this procedure are expected soon.

The consistent evolution and application of operators is a frontier for using RG in nuclear physics and there are many opportunities for ground-breaking calculations. Recent work with new operators include the calculation of the ${ }^{4}$ He total photo absorption cross section in Ref. [114, which for the first time tests the consistency of the SRG approach with a continuum observable, and the evaluation of neutrinoless doublebeta decay with SRG-evolved interactions in Ref. [115]. Adapting the RG technology developed for evolving and evaluating Hamiltonians to extend these and related studies to use fully consistent evolved operators is an important goal.

\subsection{Scale dependence of short-range correlations}

Recent experimental studies of proton knock-out reactions off nuclei at high-momentum transfer (see figure 32) have been explained by invoking short-range correlations (SRCs) 


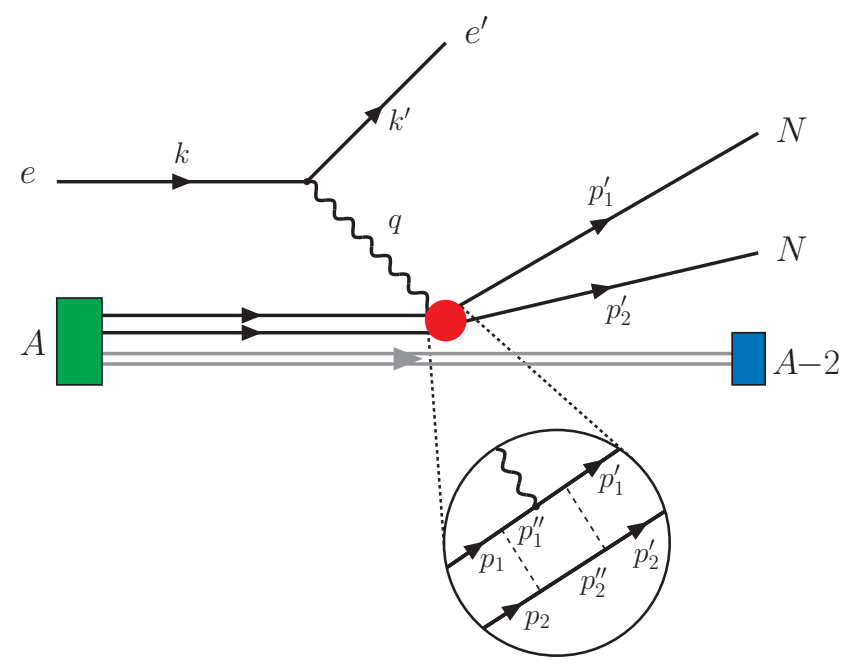

Figure 32. Illustration of different interpretations of deep-inelastic two-body knockout reactions: In the SRC picture NN interactions scatter two-body states in the initial nucleus with small initial momenta $p_{1}$ and $p_{2}$ to states with large intermediate relative momenta $p_{1}^{\prime \prime}$ and $p_{2}^{\prime \prime}$, which are then knocked out by the photon via a one-body interaction (see magnification of red vertex). The second interaction line represents a final state interaction. In general, the vertex function depends on the RG resolution scale. At low scales the wave functions are much simpler, but the vertex is a more complex two-body operator.
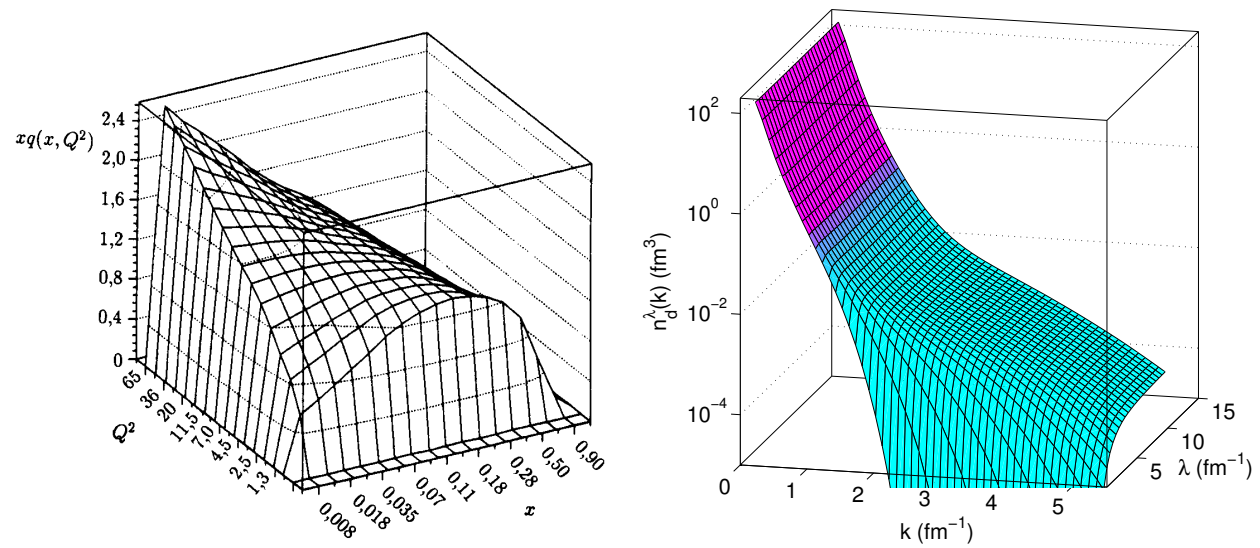

Figure 33. Quark parton distribution $x q\left(x, Q^{2}\right)$ as a function of $x$ and $Q^{2}$ (left, from [116]) and deuteron momentum distribution $n(k)$ at different SRG resolutions $\lambda$ (right).

in nuclear systems, which are manifested as enhanced strength in relative momentum distributions well above the nuclear Fermi momentum [118, 119, 120, 121]. Such explanations may seem at odds with RG evolution, which leads to many-body wave functions with highly suppressed SRCs. But the RG implies that nuclear momentum distributions are scale (and scheme) dependent, just like QCD parton distributions [107]. This analogy is illustrated in figure 33 . In the left panel, the combination $x q\left(x, Q^{2}\right)$ 


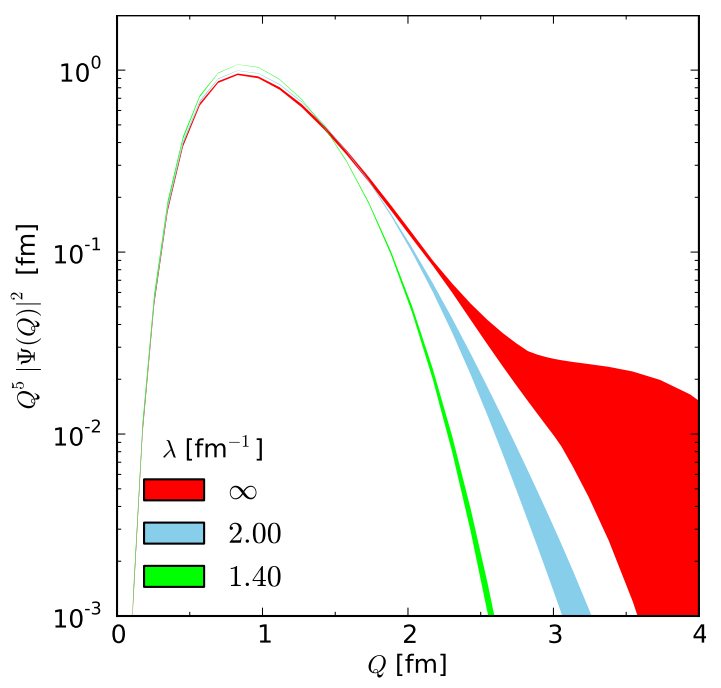

Figure 34. SRG evolution of the triton probability distribution as a function of hypermomentum $Q$ for several different SRG $\lambda$ 's for a set of chiral EFT interactions. These are plotted as bands that span the range of the wave functions from different initial 2N+3N N2LO interactions. See Ref. [26] for details.
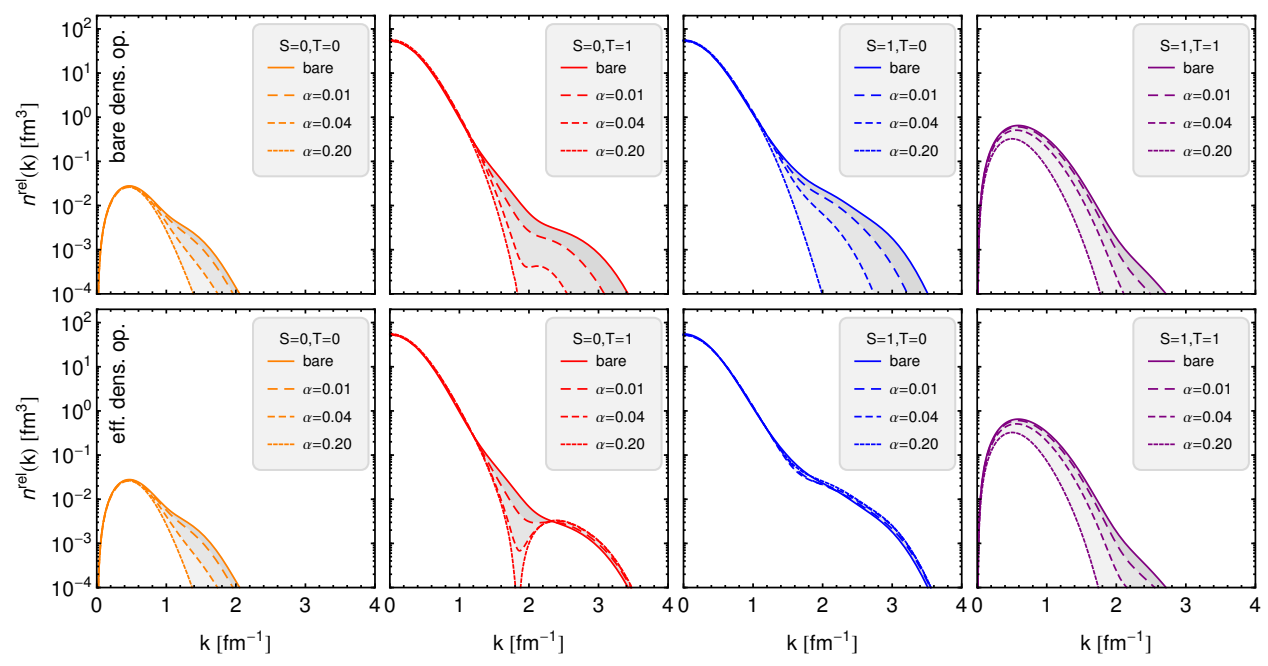

Figure 35. Two-body relative momentum distribution in ${ }^{4} \mathrm{He}$ 117.

measures the share of momentum carried by quarks in a proton within a particular $x$ interval [116]. This momentum distribution changes as a function of the resolution scale $Q^{2}$ according to $\mathrm{RG}$ evolution equations. In the right panel, the deuteron momentum distribution $n^{\lambda}(k)$ for an initial AV18 potential (the choice of potential is a scheme dependence) is SRG-evolved from $\lambda=\infty$ (corresponding to the initial potential) down to $\lambda=1.5 \mathrm{fm}^{-1}$. It is evident that the high-momentum tail, which is identified with SRC physics, is highly scale dependent and is essentially eliminated at lower resolution.

Recent calculations demonstrate the scale and scheme dependence of the momentum distribution in few-body nuclei. The probability distribution of the 
hypermomentum $Q$ of the triton is shown in figure 34 [26]. The high momentum components of the triton wave function are seen to disappear as the SRG resolution scale $\lambda$ is lowered. The spread of each band shows the scheme dependence at each $\lambda$; that is, the dependence on the initial interaction. As the interaction becomes more universal with decreasing $\lambda$, the scheme dependence naturally decreases as well. A comparison of unevolved ("bare") and evolved two-body momentum distribution in ${ }^{4} \mathrm{He}$ at four SRG resolutions is shown in figure 35, where the densities are separated into spin-isospin channels $(S$ and $T)[122,123$. The top row uses unevolved operators and illustrates the usual suppression of high-momentum components with SRG evolution. The bottom row uses operators consistently evolved but only at the two-body level, i.e., induced threebody components are dropped [117]. If all components were kept, the results would be identical at all resolutions, so the deviations from the initial distributions indicate the expected size of three-body pieces at different momenta and in different channels. For $S=1, T=0$, the result is almost unitary, while for $S=0, T=1$ deviations are limited to a range of intermediate momenta, and there are significant deviations in the other two channels. Follow-up studies will soon be possible using evolved operators with induced three-body components maintained.

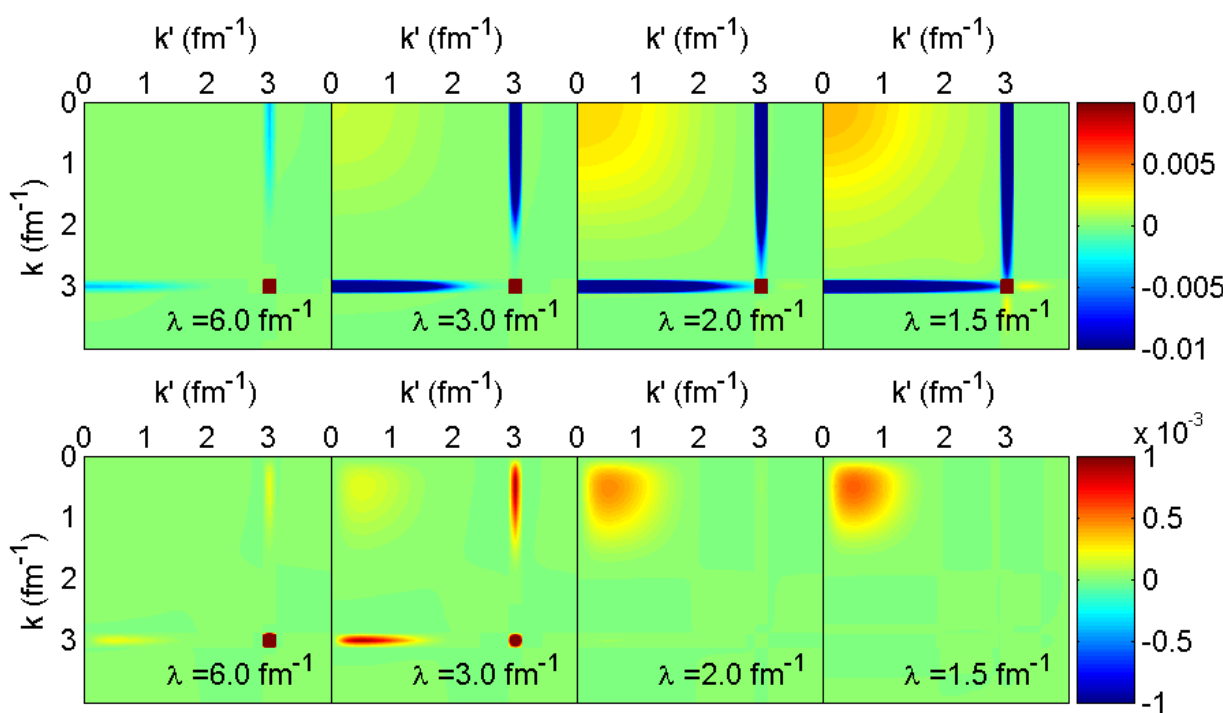

Figure 36. The top plot is the integrand of the SRG-evolved operator $a_{q}^{\dagger} a_{q}$ in the deuteron channel evaluated at $q=3 \mathrm{fm}^{-1}$. The bottom plot is the same operator but now sandwiched between the deuteron wave functions (see Ref. [113]).

If there are no high momentum components at low RG resolution, how do we interpret the physics in experiments such as in figure 32? The SRG unitary transformation approach means that cross sections should be invariant under a change in resolution. Indeed, the evolution of the wave functions describing the structure is compensated by the evolution of the operators describing the reaction. This means that the relative contributions of structure, currents, and initial/final state interactions are not fixed. A key question is then: What is the appropriate resolution scale for a given 


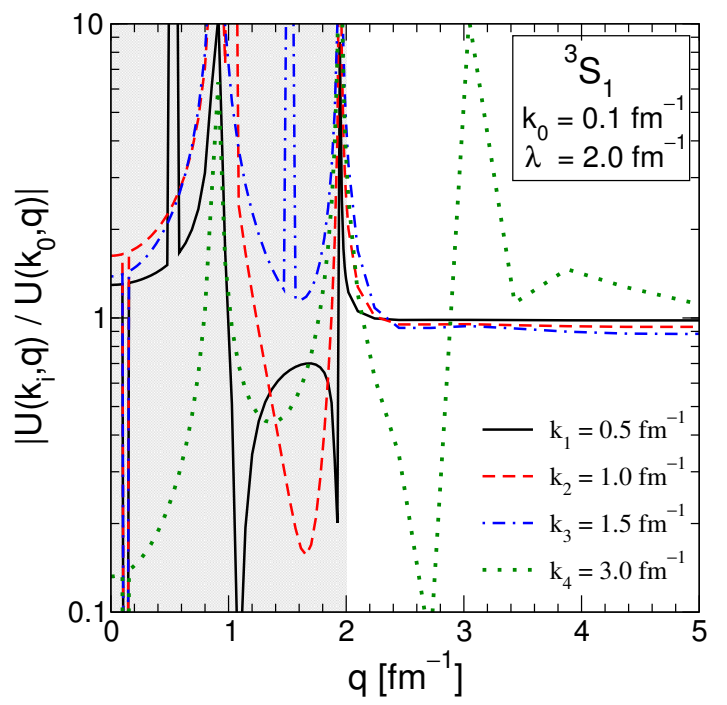

Figure 37. Ratio of two-body SRG unitary transformations $U\left(k_{i}, q\right) / U\left(k_{0}, q\right)$ at $\lambda=2.0 \mathrm{fm}^{-1}$ for the ${ }^{3} \mathrm{~S}_{1}$ channel, plotted as a function of $q$ at fixed $k_{i}$ and $k_{0}$. Factorization is signaled by a plateau in this ratio; it is expected when $q \gg \lambda$ (nonshaded region) and $k_{i} \ll \lambda$ (which is valid for $k_{1}, k_{2}$, and $k_{3}$ but not $k_{4}$ ). See Ref. [113] for details.

process; e.g., can we minimize complications such as final-state interactions?

The changing physics of operators with decreasing resolution is illustrated in figure 36. The top row shows the integrand of the simple operator $a_{q}^{\dagger} a_{q}$ at $q=3 \mathrm{fm}^{-1}$ in the deuteron channel. At high resolution $\left(\lambda=6 \mathrm{fm}^{-1}\right)$, it is essentially a one-body operator measuring the momentum strength at $k=3 \mathrm{fm}^{-1}$. At lower resolutions, the one-body part remains (one-body operators do not evolve for the usual SRG generator) but smoothly distributed strength develops at low momentum. When this operator is sandwiched in the deuteron wave function (bottom row), the contribution at $k=3 \mathrm{fm}^{-1}$ fades away at lower resolutions because the wave function completely suppresses high momentum contributions, but it is precisely replaced by the smooth low momentum contribution, reproducing the original (unevolved) momentum distribution at all resolution scales [113.

A similar trade-off of structure and reaction happens within the magnified blob in figure 32. At high resolution, the major contribution to two-nucleon knockout is when the two-body interaction couples a low-momentum pair to high momentum (creating an $\mathrm{SRC}$ in the nuclear wave function), which is subsequently knocked out by the photon via the dominant one-body interaction. At low resolution, the blob instead describes a two-body operator vertex with two soft initial momenta and two hard final momenta, which represent the observed knocked out particles.

The low-resolution picture is also accompanied by a major simplification from the scale separation of low and high-energy physics. This is manifested by a corresponding factorization of the unitary transformation, $U(k, q) \approx K(k) Q(q)$ for $k \ll \lambda$ and $q \gg \lambda$, which is demonstrated in figure 37. The factorization of the unitary transformation 
was shown in Ref. [113] to follow from effective interaction methods as well as the nonrelativistic operator product expansion [124, 125, 126]. In Ref. [127], Bogner and Roscher applied basic decoupling and scale-separation arguments to extend these results to arbitrary low-energy $A$-body states, showing that the high-momentum tails of momentum distributions and static structure factors factorize into the product of a universal function of momentum fixed by two-body physics, and a state-dependent matrix element that is sensitive only to low-momentum structure of the many-body state. This separation provides an alternative interpretation of phenomena like nuclear scaling [119, 120, 121], because the universal part will cancel (to leading order) in ratios of high-momentum tails or inclusive cross sections for different nuclei [113], leading to characteristic plateaus. The question under active investigation is whether this factorization can be exploited to quantitatively calculate nuclear scaling ratios as well as higher-order corrections.

\section{Summary and outlook}

The general strategy of applying the renormalization group ( $R G$ ) to low-energy physics is to lower the resolution of inter-nucleon interactions while tracking dependence on it. High-resolution interactions contain strong coupling of low momenta to high momenta, which complicates solutions of the many-body problem for low-energy properties. An RG evolution leads to much fewer correlated wave functions at low resolution and to faster convergence of many-body methods. Current SRG flow equations decouple low and high momenta in the form of band or block diagonalization of the Hamiltonian matrix. They represent a series of unitary transformations, in which observables are not altered but the physics interpretation can (and in general will) change. During the evolution to lower resolution non-local interactions and many-body operators are induced, which must be accommodated. In practice that means the RG evolution is performed until few-body forces start to grow rapidly, so that no controlled many-body calculations are possible anymore.

The RG technology also provides new tools to estimate theoretical uncertainties. The basic idea is that, in principle, observables should be unchanged with RG evolution, i.e., be independent of the resolution scale. In practice however, there are approximations in the $\mathrm{RG}$ implementation and in calculating nuclear observables due to truncation of "induced" many-body forces/operators and from many-body approximations. For nuclei there can be dramatic changes even with apparently small changes in the resolution scale. These resolution-scale dependences can be used as diagnostics of approximations. Recent applications of resolution-scale dependence include:

- using cutoff dependence at different orders in an EFT expansion to investigate the validity of chiral power counting, which carries over to the corresponding RGevolved interactions; 
- using the running of ground-state energies of nuclei with resolution scales to estimate errors, identify correlations and diagnosing missing many-body forces (e.g., Tjon lines) [128, 129, 32, 70, 64];

- validating MBPT convergence in calculations for infinite nuclear matter and setting lower bounds on the errors from uncertainties in many-body interactions [23, 55, 41];

- identifying and characterizing scheme-dependent observables, such as spectroscopic factors and effective single-particle energies [108, 109, 107].

In this review, we have shown glimpses of the many promising applications of RG methods to nuclei. Configuration interaction, coupled cluster, IM-SRG, and self-consistent Green's function approaches using softened interactions converge faster, opening up new possibilities to extend the limits of computational feasibility. Ground-breaking ab-initio reaction calculations are now possible. Applications of lowmomentum interactions to microscopic shell model calculations bring new understanding to phenomenological results, highlighting the role of three-body forces. In-medium SRG offers a means to directly calculate effective shell-model interactions. Because manybody perturbation theory (possibly resummed) is feasible with the evolved interactions, the door is opened to constructive nuclear density functional theory [130, 131, 132, 133, 97, 40.

Despite many successes, there are also open questions and difficult problems in applying RG to low-energy nuclear physics. Here is a subset:

- Perhaps the most outstanding issue at present is the size and nature of four-body contributions in larger nuclei. Calculations of induced four-body forces are now feasible and should provide direct tests in the near future.

- More generally, we need quantitative power counting for evolved many-body operators. That is, how do we anticipate the size of contributions from induced many-body interactions and other operators? This is essential if we are to have reliable estimates of theoretical errors, because truncations are unavoidable. We need both analytic estimates to guide us as well as more extensive numerical tests. Many of the same issues apply to chiral EFT; can the additional information available from SRG flow parameter dependence help with analyzing or even constructing EFT's?

- In Sections 2.2 and 4.1 we presented calculations for infinite nuclear matter and finite nuclei using different types of MBPT based on RG-evolved interactions. How can the different convergence patterns be reconciled and what is the best measure to quantify the perturbativeness of nuclear interactions?

- Only a few possibilities for SRG generators have been considered so far for nuclear systems. Can other choices for the SRG $G_{s}$ operator help to control the growth of many-body forces? Can a generator be found to drive non-local potentials to a more local form, so they can be used with quantum Monte Carlo methods? Or can the SRG equations be formulated to directly produce a local projection and a perturbative residual interaction? 
- An apparent close connection between the block-diagonal generator SRG and the "standard" $V_{\text {low } k}$ RG has been established empirically, but a formal demonstration of the connection and its limits has not been made.

- Are there other bases for SRG evolution that would be advantageous? Recent momentum-space implementations will provide necessary checks of evolution in the harmonic oscillator basis, and the evolved interactions in this form will be directly applied to test MBPT in infinite matter and to test nuclear scaling. The brand new use of hyperspherical coordinates [26] should be particularly useful for visualization of many-body forces.

- There are many open questions and problems involving operators. These include formal issues such as the scaling of many-body operators and technical issues such as how to handle boosts of operators that are not galilean invariant. And there are simply many applications that are in there infancy (e.g., electroweak processes).

- The flow to universal form exhibited by two-body interactions has been clear from the beginning of RG applications to nuclei. The nature of this behavior for manybody interactions or for other operators is an still open question, although under active investigation.

- How does the SRG relate to functional RG equations and field theory methods?

- How can we use more of the power of the RG itself?

Developments involving renormalization group methods in low-energy nuclear physics are occurring at a rapid pace, so we can expect steady progress on these challenges and opportunities.

\section{Acknowledgments}

We thank S. Bogner, A. Dyhdalo, A. Gezerlis, H. Hergert, J. Menéndez, S. More, R. Perry, R. Roth, A. Schwenk, V. Somà, M. Voskresenskaya, and K. Wendt for helpful discussions and comments. This work was supported in part by the National Science Foundation under Grant No. PHY-1002478, the U.S. Department of Energy under Grant No. de-sc0008533 (SciDAC-3/NUCLEI project), and an award of computational resources from the Ohio Supercomputer Center.

\section{References}

[1] Erler J, Birge N, Kortelainen M, Nazarewicz W, Olsen E, Perhac A M and Stoitsov M 2012 Nature $\mathbf{4 8 6} 509-512$

[2] 2007 DOE/NSF Nuclear Science Advisory Committe, The Frontiers of Nuclear Science: A LongRange Plan

[3] Aoki S, Hatsuda T and Ishii N 2008 Comput. Sci. Dis. 1015009

[4] Bogner S K, Furnstahl R J and Schwenk A 2010 Prog. Part. Nucl. Phys. 65 94-147

[5] Ring P and Schuck P 2005 The Nuclear Many-Body Problem (Berlin: Springer)

[6] Preston M and Bhaduri R 1975 Structure of the Nucleus (Reading, MA: Addison-Wesley)

[7] Navratil P, Quaglioni S, Stetcu I and Barrett B R 2009 J. Phys. G 36083101 
[8] Guth A H, Huang K and Jaffe R L (eds) 1983 Asymptotic Realms of Physics (Cambridge, MA: MIT Press)

[9] Furnstahl R 2012 Nucl. Phys. Proc. Suppl. 228 139-175

[10] Bogner S K, Kuo T T S and Schwenk A 2003 Phys. Rept. 386 1-27

[11] Peskin M and Schroeder D 1995 An Introduction to Quantum Field Theory (Reading, MA: Addison-Wesley Publishing Company)

[12] Wegner F 1994 Ann. Phys. 50677

[13] Wegner F J 2000 Nucl. Phys. Proc. Suppl. 90 141-146

[14] Wegner F J 2006 Journal of Physics A: Mathematical and General 39 8221-8230

[15] Glazek S D and Wilson K G 1993 Phys. Rev. D 48 5863-5872

[16] Bogner S K, Furnstahl R J and Perry R J 2007 Phys. Rev. C 75061001

[17] Wendt K, Furnstahl R and Ramanan S 2012 Phys. Rev. C 86014003

[18] Glazek S D and Perry R J 2008 Phys. Rev. D 78045011

[19] Wendt K, Furnstahl R and Perry R 2011 Phys. Rev. C 83034005

[20] Entem D R and Machleidt R 2003 Phys. Rev. C 68041001

[21] Anderson E et al. 2008 Phys. Rev. C 77037001

[22] Li W, Anderson E and Furnstahl R 2011 Phys. Rev. C 84054002

[23] Hebeler K, Bogner S, Furnstahl R, Nogga A and Schwenk A 2011 Phys. Rev. C 83031301

[24] Bender M, Heenen P H and Reinhard P G 2003 Rev. Mod. Phys. 75 121-180

[25] Kaiser N 2012 Eur. Phys. J. A 48135

[26] Wendt K A 2013 arXiv:1304.1431

[27] Jurgenson E D, Navratil P and Furnstahl R J 2009 Phys. Rev. Lett. 103082501

[28] Epelbaum E, Hammer H W and Meißner U G 2009 Rev. Mod. Phys. 81 1773-1825

[29] Hammer H W, Nogga A and Schwenk A 2013 Rev. Mod. Phys. 85197

[30] Jurgenson E, Navratil P and Furnstahl R 2011 Phys. Rev. C 83034301

[31] Roth R, Langhammer J, Calci A, Binder S and Navratil P 2011 Phys. Rev. Lett. 107072501

[32] Roth R, Binder S, Vobig K, Calci A, Langhammer J et al. 2012 Phys. Rev. Lett. 109052501

[33] Otsuka T, Suzuki T, Holt J D, Schwenk A and Akaishi Y 2010 Phys. Rev. Lett. 105032501

[34] Holt J D, Otsuka T, Schwenk A and Suzuki T 2012 J. Phys. G 39085111

[35] Hebeler K and Schwenk A 2010 Phys. Rev. C 82014314

[36] Hebeler K 2012 Phys. Rev. C 85021002

[37] Epelbaum E 2006 Prog. Part. Nucl. Phys. 57 654-741

[38] Bernard V, Epelbaum E, Krebs H and Meißner U G 2008 Phys. Rev. C 77064004

[39] Bernard V, Epelbaum E, Krebs H and Meißner U G 2011 Phys. Rev. C 84054001

[40] Holt J, Kaiser N and Weise W 2013 arXiv:1304.6350

[41] Gezerlis A, Tews I, Epelbaum E, Gandolfi S, Hebeler K et al. 2013 arXiv 1303.6243

[42] Giorgini S, Pitaevskii L P and Stringari S 2008 Rev. Mod. Phys. 80 1215-1274

[43] Haensel P, Potekhin A and Yakovlev D G 2006 Springer

[44] Akmal A, Pandharipande V R and Ravenhall D G 1998 Phys. Rev. C 58 1804-1828

[45] Lejeune A, Lombardo U and Zuo W 2000 Phys. Lett. B 47745

[46] Carlson J, Chang S Y, Pandharipande V R and Schmidt K E 2003 Phys. Rev. Lett. 91050401

[47] Gezerlis A and Carlson J 2008 Phys. Rev. C 77032801

[48] Schwenk A and Pethick C J 2005 Phys. Rev. Lett. 95160401

[49] Steiner A W, Prakash M, Lattimer J M and Ellis P J 2005 Phys. Rept. 411 325-375

[50] Hebeler K, Lattimer J, Pethick C and Schwenk A 2013 arXiv:1303.4662

[51] Read J S, Markakis C, Shibata M, Uryu K, Creighton J D et al. 2009 Phys. Rev. D 79124033

[52] Hebeler K, Lattimer J, Pethick C and Schwenk A 2010 Phys. Rev. Lett. 105161102

[53] Demorest P, Pennucci T, Ransom S, Roberts M and Hessels J 2010 Nature 467 1081-1083

[54] Krüger T, Tews I, Hebeler K and Schwenk A 2013 arXiv 1304.2212

[55] Hebeler K and Furnstahl R 2013 Phys. Rev. C 87031302

[56] Roth R and Langhammer J 2010 Phys. Lett. B 683 272-277 
[57] Langhammer J, Roth R and Stumpf C 2012 Phys. Rev. C 86054315

[58] Hjorth-Jensen M, Kuo T T S and Osnes E 1995 Phys. Rept. 261 125-270

[59] Holt J, Menendez J and Schwenk A 2013 Eur. Phys. J. A 4939

[60] Gallant A, Bale J, Brunner T, Chowdhury U, Ettenauer S et al. 2012 Phys. Rev. Lett. 109032506

[61] Holt J, Menendez J and Schwenk A 2013 Phys. Rev. Lett. 110022502

[62] Holt J, Menendez J and Schwenk A 2013 arXiv:1304.0434

[63] Tsukiyama K, Bogner S and Schwenk A 2012 Phys. Rev. C 85061304

[64] Jurgenson E, Maris P, Furnstahl R, Navratil P, Ormand W et al. 2013 arXiv:1302.5473

[65] Roth R 2009 Phys. Rev. C 79064324

[66] Kruse M, Jurgenson E, Navrtil P, Barrett B and Ormand W 2013 Phys. Rev. C 87044301

[67] Hagen G, Dean D J, Hjorth-Jensen M, Papenbrock T and Schwenk A 2007 Phys. Rev. C 76 044305

[68] Hagen G et al. 2007 Phys. Rev. C 76034302

[69] Hagen G, Papenbrock T, Dean D and Hjorth-Jensen M 2010 Phys. Rev. C 82034330

[70] Binder S, Langhammer J, Calci A, Navratil P and Roth R 2013 Phys. Rev. C 87 021303(R)

[71] Navratil P, Roth R and Quaglioni S 2011 Phys. Lett. B 704 379-383

[72] Furnstahl R J 2011 Nuclear Physics News 21 18-24

[73] Nam H, Stoitsov M, Nazarewicz W, Bulgac A, Hagen G et al. 2012 J. Phys. Conf. Ser. 402 012033

[74] Bogner S, Bulgac A, Carlson J A, Engel J, Fann G et al. 2013 arXiv 1304.3713

[75] Navratil P, Quaglioni S and Roth R 2011 J. Phys. Conf. Ser. 312082002

[76] Navratil P, Roth R and Quaglioni S 2010 Phys. Rev. C 82034609

[77] Navratil P and Quaglioni S 2012 Phys. Rev. Lett. 108042503

[78] Quaglioni S, Navratil P, Hupin G, Langhammer J, Romero-Redondo C et al. 2012 arXiv:1210. 2020

[79] Baroni S, Navratil P and Quaglioni S 2013 Phys. Rev. C 87034326

[80] Roth R, Neff T and Feldmeier H 2010 Prog. Part. Nucl. Phys. 65 50-93

[81] Neff T 2012 J. Phys. Conf. Ser. 403012028

[82] Neff T 2011 Phys. Rev. Lett. 106042502

[83] Neff T, Feldmeier H and Langanke K 2010 PoS NICXI 017

[84] Tsukiyama K, Bogner S and Schwenk A 2011 Phys. Rev. Lett. 106222502

[85] Hergert H, Bogner S, Binder S, Calci A, Langhammer J et al. 2013 Phys. Rev. C 87034307

[86] Kehrein S 2006 The Flow Equation Approach to Many-Particle Systems (Berlin: Springer)

[87] Hergert H, Binder S, Calci A, Langhammer J and Roth R 2013 Phys. Rev. Lett. (in press)

[88] Audi G, Wapstra A and Thibault C 2002 Nucl. Phys. A 729 337-676

[89] Shavitt I and Bartlett R 2009 Many-Body Methods in Chemistry and Physics: MBPT and Coupled-Cluster Theory (Cambridge University Press)

[90] Leidemann W and Orlandini G 2012 arXiv 1204.4617

[91] Barrett B R, Navratil P and Vary J P 2013 Prog. Part. Nucl. Phys. 69 131-181

[92] Bacca S, Barnea N and Schwenk A 2012 Phys. Rev. C 86034321

[93] Papadimitriou G, Rotureau J, Michel N, Ploszajczak M and Barrett B 2013 arXiv 1301.7140

[94] Rotureau J, Michel N, Nazarewicz W, Ploszajczak M and Dukelsky J 2009 Phys. Rev. C 79 014304

[95] Pigg D, Hagen G, Nam H and Papenbrock T 2012 Phys. Rev. C 86014308

[96] Soma V, Duguet T and Barbieri C 2011 Phys.Rev. C84 064317

[97] Duguet T 2012 arXiv 1204.2737

[98] Barbieri C, Cipollone A, Soma V, Duguet T and Navratil P 2012 arXiv: 1211.3315

[99] Soma V, Barbieri C and Duguet T 2013 Phys. Rev. C 87011303

[100] Cipollone A, Barbieri C and Navrtil P 2013 arXiv 1303.4900

[101] Coon S A, Avetian M I, Kruse M K, van Kolck U, Maris P et al. 2012 Phys. Rev. C 86054002

[102] Furnstahl R, Hagen G and Papenbrock T 2012 Phys. Rev. C 86031301 
[103] More S, Ekstrom A, Furnstahl R, Hagen G and Papenbrock T 2013 Phys. Rev. C 87044326

[104] Lynn J and Schmidt K 2012 Phys. Rev. C 86014324

[105] Launey K D, Dytrych T c v and Draayer J P 2012 Phys. Rev. C 85044003

[106] Timoteo V, Szpigel S and Ruiz Arriola E 2012 Phys.Rev. C86 034002

[107] Furnstahl R J and Schwenk A 2010 J. Phys. G 37064005

[108] Jensen O, Hagen G, Papenbrock T, Dean D and Vaagen J 2010 Phys. Rev. C 82014310

[109] Duguet T and Hagen G 2012 Phys. Rev. C 85034330

[110] Bogner S K, Furnstahl R J, Ramanan S and Schwenk A 2007 Nucl. Phys. A 784 79-103

[111] Jurgenson E D, Bogner S K, Furnstahl R J and Perry R J 2008 Phys. Rev. C 78014003

[112] Holt J D and Engel J 2013 arXiv 1304.4202

[113] Anderson E, Bogner S, Furnstahl R and Perry R 2010 Phys. Rev. C 82054001

[114] Schuster M D, Quaglioni S, Johnson C W, Jurgenson E D and Navratil P 2013 arXiv: 1304.5491

[115] Shukla D, Engel J and Navratil P 2011 Phys. Rev. C 84044316

[116] Povh B, Rith K, Scholz C and Zetsche F 2004 Particles and nuclei: an introduction to the physical concepts; 4th ed. (Berlin: Springer)

[117] Neff T Private communication

[118] Subedi R, Shneor R, Monaghan P, Anderson B, Aniol K et al. 2008 Science 320 1476-1478

[119] Frankfurt L, Sargsian M and Strikman M 2008 Int. J. Mod. Phys. A 23 2991-3055

[120] Arrington J, Higinbotham D, Rosner G and Sargsian M 2012 Prog. Part. Nucl. Phys. 67 898-938

[121] Alvioli M, Ciofi degli Atti C, Kaptari L, Mezzetti C and Morita H 2013 Phys. Rev. C 87034603

[122] Horiuchi W, Feldmeier H, Neff T and Suzuki Y 2013 Few Body Syst. 54 279-282

[123] Feldmeier H, Horiuchi W, Neff T and Suzuki Y 2011 Phys. Rev. C 84054003

[124] Lepage G 1997 arXiv:nucl-th/9706029

[125] Braaten E and Platter L 2008 Phys. Rev. Lett. 100205301

[126] Braaten E, Kang D and Platter L 2008 Phys. Rev. A 78053606

[127] Bogner S and Roscher D 2012 Phys. Rev. C 86064304

[128] Hammer H W and Platter L 2010 Ann.Rev.Nucl.Part.Sci. 60 207-236

[129] Hammer H W and Platter L 2011 Phil.Trans.Roy.Soc.Lond. A369 2679

[130] Bogner S K, Furnstahl R J and Platter L 2009 Eur. Phys. J. A 39 219-241

[131] Drut J E, Furnstahl R J and Platter L 2010 Prog. Part. Nucl. Phys. 64120

[132] Duguet T, Lesinski T, Hebeler K and Schwenk A 2010 Mod. Phys. Lett. A25 1989-1992

[133] Stoitsov M, Kortelainen M, Bogner S, Duguet T, Furnstahl R et al. 2010 Phys. Rev. C 82054307 